\title{
Two necessary conditions for strategy-proofness: On what domains are they also sufficient?
}

\author{
Salvador Barberà ${ }^{\mathrm{a}, \mathrm{b}, *}$, Dolors Berga ${ }^{\mathrm{c}}$, Bernardo Moreno ${ }^{\mathrm{d}}$ \\ a MOVE, Universitat Autònoma de Barcelona, and Barcelona GSE, Spain \\ b Departament d'Economia i d'Història Econòmica, Edifici B, 08193 Bellaterra, Spain \\ c Departament d'Economia, Campus de Montilivi, Universitat de Girona, 17071 Girona, Spain \\ d Departamento de Teoría e Historia Económica, Facultad de Ciencias Económicas y Empresariales, Campus de El Ejido, 29071 Málaga, Spain
}

\section{A R T I C L E I N F O}

\section{Article history:}

Received 4 August 2011

Available online 2 March 2012

\section{JEL classification:}

D71

Keywords:

Strategy-proofness

Reshuffling invariance

Monotonicity

Intertwined domains

\begin{abstract}
A B S T R A C T
A social choice function may or may not satisfy a desirable property depending on its domain of definition. For the same reason, different conditions may be equivalent for functions defined on some domains, while not in other cases. Understanding the role of domains is therefore a crucial issue in mechanism design. We illustrate this point by analyzing the role of two conditions, monotonicity and reshuffling invariance, that are always necessary, but not always sufficient for strategy-proofness. We identify domains, that we call intertwined, ensuring the equivalence between these two conditions and that of strategy-proofness for whatever functions are defined on them. We prove that some important domains are intertwined: those of single-peaked preferences, both with public and private goods, and also those arising in simple models of house allocation. We also discuss the relationship between our conditions on functions and domains with others considered in the literature.
\end{abstract}

(c) 2012 Elsevier Inc. All rights reserved.

\section{Introduction}

Strategy-proofness is a highly desirable but hard to meet property of social choice functions. Efforts to understand the implications of this requirement have naturally led to study properties that are necessary and/or sufficient for its fulfillment (Muller and Satterthwaite, 1977; Moulin, 1988). Efforts to identify conditions under which it can be satisfied by non-trivial rules have directed attention to the importance of the domains on which they are defined (Moulin, 1980; Barberà et al., 1991, 1993; Sprumont, 1991; Moulin and Shenker, 1992; Pápai, 2000, etc.).

We concentrate here in the study of two conditions that are clearly related with that of strategy-proofness and that we find especially attractive. We call them reshuffling invariance and monotonicity. We first show that they are jointly equivalent to strategy-proofness for social choice functions defined on the universal domain. Then we remark that the equivalence

\footnotetext{
We thank Lars Ehlers and James Schummer for very useful comments on an earlier version of the paper. We also thank two referees for their insightful comments, that motivated us to get deeper into the most delicate aspects of the problem we are dealing with, and of the solutions we propose. Salvador Barberà gratefully acknowledges support from the Spanish Ministry of Science and Innovation through grant "Consolidated Group-C" ECO2008-04756, from the Generalitat de Catalunya, Departament d'Universitats, Recerca i Societat de la Informació through the Distinció per a la Promoció de la Recerca Universitària and grant SGR2009-0419. Dolors Berga acknowledges the support of the Spanish Ministry of Science and Innovation through grants SEJ200760671 and ECO2010-16353, of Generalitat de Catalunya, through grants 2010-BE-00608 and SGR2009-0189. She also acknowledges the Research Recognition Programme of the Barcelona GSE. Bernardo Moreno gratefully acknowledges financial support from Junta de Andalucia through grants SEJ4941 and SEJ-5980 and the Spanish Ministry of Science and Technology through grant ECO2008-03674.

* Corresponding author at: Departament d'Economia i d'Història Econòmica, Edifici B, 08193 Bellaterra, Spain.

E-mail addresses: salvador.barbera@uab.cat (S. Barberà), dolors.berga@udg.edu (D. Berga), bernardo@uma.es (B. Moreno).
} 
between our conditions and strategy-proofness does not hold in general. Reshuffling invariance and monotonicity are always necessary for strategy-proofness, whatever the domain of definition of the functions, but need not be sufficient. Because of that, we ask ourselves the following question: can we identify domains of preferences having the property that, when functions are defined on these domains, then our conditions are equivalent to strategy-proofness?

We answer this question in the positive. For those domains that we call intertwined, and for any possible rule defined on them, the equivalence holds.

Other authors have proposed alternative conditions, also closely related to strategy-proofness. We single out two of them, for reference and comparison: Moulin's (1988) notion of strong monotonicity and Muller and Satterthwaite's (1977) strong positive association. These conditions are also equivalent to strategy-proofness, and therefore to the ones we propose, for functions defined on the universal domain of strict preferences. ${ }^{1}$ We show that, indeed, the equivalence still holds for any functions defined on any intertwined domain, but not necessarily otherwise.

This research is part of an effort to understand the role of domain restrictions in mechanism design, and more specifically in connection with the possibility of achieving strategy-proofness. Since the bite of this and other related conditions, like those we introduce in the present paper, depends on the type of preferences that are admissible for individuals, it is natural that they precipitate impossibility results for some domains, and not for others.

In fact, the equivalence of different sets of formal conditions is not particularly illuminating when these apply to social choice functions defined on the universal domain, since the only rules that can satisfy them in that case and have more than two alternatives in the range must be dictatorial. The comparison between different requirements becomes much more significant when these are imposed on domains on which it is indeed possible to find non-trivial rules that may be able to meet some of the properties.

It is interesting to notice that some of the restricted domains admitting non-trivial strategy-proof social choice functions are indeed intertwined. This is the case, for example, if our domain is the set of all strict preference profiles that are singlepeaked (for a given order of the alternatives). It is also the case when alternatives are allocations of a single indivisible object to agents who only care about the object they receive, like in simple models of house allocation. Yet, other domains also admitting non-trivial strategy-proof rules, like those where each agent exhibits separable or additive preferences, are not intertwined.

The notion of group strategy-proofness is stronger, and in a sense more natural and attractive than that of (individual) strategy-proofness. Yet, in view of the difficulty to identify functions satisfying the weaker of the two properties, the analysis of the stronger one has been less intensive. One of our results in this paper reinforces our understanding of the connections between the two versions of strategy-proofness, and also between those and the remaining properties under discussion: reshuffling invariance, monotonicity, strong monotonicity or strong positive association. When the set of admissible preferences is identical for all agents, then intertwined domains also satisfy the property of indirect sequential inclusion. And, as shown in Barberà et al. (2010), this means that any function defined on these domains will be group strategy-proof if and only if it is strategy-proof. As a corollary, we learn that the conditions that were till now associated with the weak notion of strategy-proofness are also necessary and sufficient for the stronger notion, on a wide variety of cases.

The structure of the paper is as follows. In Section 2 we define the framework, we introduce different properties of social choice functions and start discussing the relationships among them. In Section 3 we present our new definition of intertwined domains and prove our main equivalence result. Section 4 shows that a number of interesting domains are indeed intertwined. Sections 5 and 6 (and Appendix A) relate our work with other parts of the literature. In Section 5 we relate our main conditions on social choice functions with others that have been discussed and used by other authors. In Section 6 and in Appendix A we compare intertwined domains with others that have been proposed over the years, to essentially conclude that our new condition is different from existing ones. Section 7 concludes.

\section{The setup: definitions and preliminary results}

Let $A$ be a finite set of alternatives ${ }^{2}$ and $N=\{1, \ldots, n\}$ be a finite set of agents. Let $\mathcal{R}$ be the set of all preorders (complete, reflexive, and transitive binary relations) on $A$ and $\mathcal{R}_{i} \subseteq \mathcal{R}$ be the set of admissible preferences for agent $i \in N$. Denote by $\mathcal{P} \subseteq \mathcal{R}$ the set of all antisymmetric preorders. We denote by $R_{i} \in \mathcal{R}_{i}$ an admissible preference relation and let as usual, $P_{i}$ and $I_{i}$ be the strict and the indifference part of $R_{i}$, respectively. When all the admissible preferences for individual $i$ are strict, we will use the notation $\mathcal{P}_{i}$, instead of the general expression $\mathcal{R}_{i}$. A preference profile, denoted by $R_{N}=$ $\left(R_{1}, \ldots, R_{n}\right)$, is an element of $X_{i \in N} \mathcal{R}_{i}$. Let $C, S \subseteq N$ be two coalitions such that $C \subseteq S$. We will write the subprofile $R_{S}=$ $\left(R_{C}, R_{S \backslash C}\right) \in \mathrm{X}_{i \in S} \mathcal{R}_{i}$ when we want to stress the role of coalition $C$ in $S$. Then the subprofiles $R_{C} \in \mathrm{X}_{i \in C} \mathcal{R}_{i}$ and $R_{S \backslash C} \in$ $\mathrm{X}_{i \in S \backslash C} \mathcal{R}_{i}$ denote the preferences of agents in $C$ and in $S \backslash C$, respectively. In the case, where we denote full preference profile (that is, when $S=N)$, we simplify notation by using $\left(R_{C}, R_{N \backslash C}\right)$ as $\left(R_{C}, R_{-C}\right)$.

\footnotetext{
1 These are not the only conditions related to strategy-proofness that are mentioned in the literature. For example, Maskin monotonicity (see Maskin, $1985,1999)$, an essential condition in the implementation literature, is equivalent to strong positive association when individual preferences are strict. But we do not insist on it, as it is not necessary for strategy-proofness in the presence of indifferences, in our discrete setting.

2 As soon as we go to a continuum of alternatives we are typically led to further complicate our simple models, to establish some topology on the set of alternatives, to introduce notions of continuity in the preferences, etc. We avoid any complications of this kind by sticking to the finite framework.
} 
For any $R_{i} \in \mathcal{R}_{i}$ and $x \in A, E\left(R_{i}, x\right)=\left\{y \in A: x I_{i} y\right\}$ stands for the indifference class to which $x$ belongs to. For any $R_{i} \in \mathcal{R}_{i}$ and $B \subseteq A$ such that for all $z, t \in B, z I_{i}$ t, define the lower contour set of $R_{i}$ at $B$ and the upper contour set of $R_{i}$ at $B$ as $L\left(R_{i}, B\right)=\left\{y \in A: x R_{i} y\right.$ for some $\left.x \in B\right\}$ and $U\left(R_{i}, B\right)=\left\{y \in A: y R_{i} x\right.$ for some $\left.x \in B\right\}$, respectively. Similarly, define the strict lower contour set at $B$ and the strict upper contour set at $B$ as $\bar{L}\left(R_{i}, B\right)=\left\{y \in A: x P_{i} y\right.$ for some $\left.x \in B\right\}$ and $\bar{U}\left(R_{i}, B\right)=$ $\left\{y \in A: y P_{i} x\right.$ for some $\left.x \in B\right\}$, respectively.

A social choice function (or also a rule) is a function $f: X_{i \in N} \mathcal{R}_{i} \rightarrow A$.

We now introduce some interesting properties that social choice functions may or may not be able to satisfy. Notice that the domains of the functions are an important part of our definitions. Indeed, a property may be satisfied by a rule on a domain, but may not when its domain is expanded or reduced.

We will focus on rules that are non-manipulable by a single agent. We first define what we mean by a manipulation and then we introduce the well-known concept of strategy-proofness.

Definition 1. A social choice function $f$ is manipulable on $\chi_{i \in N} \mathcal{R}_{i}$ at $R_{N} \in X_{i \in N} \mathcal{R}_{i}$ if there exists an agent $i \in N$ and $R_{i}^{\prime} \in \mathcal{R}_{i}\left(R_{i}^{\prime} \neq R_{i}\right)$ such that $f\left(R_{i}^{\prime}, R_{-i}\right) P_{i} f\left(R_{N}\right)$. We say that agent $i$ manipulates $f$ at $R_{N}$ via $R_{i}^{\prime}$.

Definition 2. A social choice function $f$ is strategy-proof on $\times_{i \in N} \mathcal{R}_{i}$ if $f$ is not manipulable at any $R_{N} \in \mathrm{X}_{i \in N} \mathcal{R}_{i}$

Notice that the domains of our social choice functions will always have the form of a cartesian product. This is necessary to give meaning to our definition of strategy-proofness. Also notice that, although the notion of a domain is attached to that of a given function, we shall also refer to any cartesian family of preference profiles as a domain, and to its cartesian subsets as its subdomains. This is consistent with tradition, although it would be more precise to call them potential domains, as we shall in fact consider sets of functions that could be defined on them.

We now define the main conditions in our characterization result, monotonicity and reshuffling invariance. Before that, an important comment is in order: All of our definitions (also the one of intertwined domains in Section 3) become nicely simplified in the case of strict preferences. For the sake of compactness we only present them in their general form. However, the interested reader is invited to consider how they would specialize when indifferences would not be allowed in our domains. Then, conditions become even more transparent. As always, introducing indifferences provides generality at the cost of added complexities.

Definition 3. Let $R_{i} \in \mathcal{R}_{i}, x \in A$. We say that $R_{i}^{\prime} \in \mathcal{R}_{i}$ is an $x$-monotonic transformation of $R_{i}$ if there exist $B_{x}^{R_{i}} \subseteq E\left(R_{i}, x\right)$, $x \in B_{x}^{R_{i}}$ such that the following conditions hold:

(i) for any $z \in B_{x}^{R_{i}}, E\left(R_{i}^{\prime}, z\right)=B_{x}^{R_{i}}$,

(ii) for any $z \in B_{x}^{R_{i}}$, for any $y \in A \backslash B_{x}^{R_{i}},\left[z R_{i} y \Rightarrow z P_{i}^{\prime} y\right]$, and

(iii) for any $y, w \in A \backslash B_{x}^{R_{i}},\left[y R_{i} w \Leftrightarrow y R_{i}^{\prime} w\right]$.

In words: $R_{i}^{\prime}$ is an $x$-monotonic transformation of $R_{i}$ if there exists a set $B_{x}^{R_{i}}$ containing $x$ which is now an indifference class of $R_{i}^{\prime}$ on its own, which was a subset of $x$ 's indifference class in $R_{i}$, and such that the relative position of its elements has improved when going from $R_{i}$ to $R_{i}^{\prime}$, while all the rest of alternatives keep in the same relative positions with each other.

We can now define monotonicity. Fig. 1 provides an illustration of how it works.

Definition 4. A social choice function $f$ satisfies monotonicity on $\mathrm{X}_{i \in N} \mathcal{R}_{i}$ if and only if for any $R_{N} \in \mathrm{X}_{i \in N} \mathcal{R}_{i}$ such that $f\left(R_{N}\right)=x$, and for any $\left(R_{i}^{\prime}, R_{-i}\right) \in \mathrm{X}_{i \in N} \mathcal{R}_{i}$ such that $R_{i}^{\prime}$ is an $x$-monotonic transformation of $R_{i}, f\left(R_{i}^{\prime}, R_{-i}\right) \in E\left(R_{i}, x\right)$.

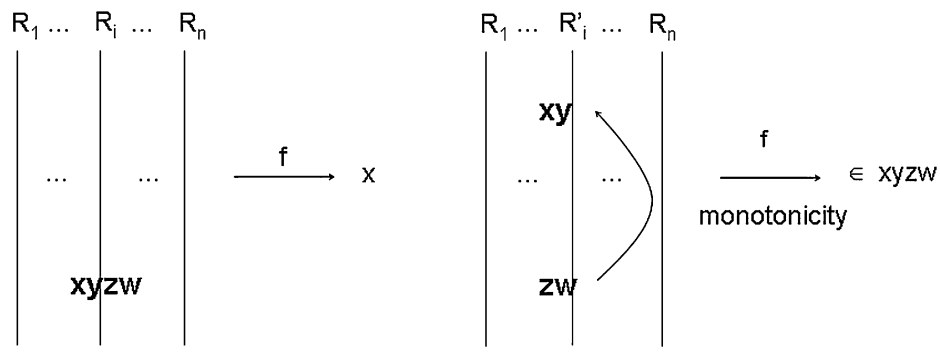

Fig. 1. Monotonicity of $f$. 
In words: If an alternative $x$ is chosen by a social choice function $f$ at profile $\left(R_{i}, R_{-i}\right)$, and $R_{i}^{\prime}$ is a new preference where $x$ has improved its position (maybe in the company of some other alternatives in his $R_{i}$ indifference class), then $f$ must still choose $x$ or some of the alternatives that were initially indifferent to it. Fig. 1 illustrates the meaning of the condition.

We now turn to our second condition. First, define reshufflings.

Definition 5. Let $R_{i} \in \mathcal{R}_{i}$ and $x \in A$. We say that $R_{i}^{\prime} \in \mathcal{R}_{i}$ is an $x$-reshuffling of $R_{i}$ if (i) $\bar{L}\left(R_{i}, x\right)=\bar{L}\left(R_{i}^{\prime}, x\right)$, and (ii) $\bar{U}\left(R_{i}^{\prime}, x\right) \subseteq$ $\bar{U}\left(R_{i}, x\right){ }^{3}$

In words: $R_{i}^{\prime}$ is an $x$-reshuffling of $R_{i}$ if it results from keeping all alternatives that were worse than $x$, as still being worse, though maybe in a different order; and all alternatives that are better than $x$ in $R_{i}^{\prime}$ were already better in $R_{i}$, again maybe in a different order. Notice that we allow some elements ranked above $x$ in $R_{i}$ to become indifferent to $x$ in $R_{i}^{\prime}$. When preferences are strict, condition (i) implies condition (ii), and furthermore, $\bar{U}\left(R_{i}^{\prime}, x\right)=\bar{U}\left(R_{i}, x\right)$.

Fig. 2 illustrates the reshuffling invariance property, which we now formally define.

Definition 6. A social choice function $f$ satisfies reshuffling invariance on $X_{i \in N} \mathcal{R}_{i}$ if and only if for any $R_{N} \in X_{i \in N} \mathcal{R}_{i}$ such that $f\left(R_{N}\right)=x$, and for any $\left(R_{i}^{\prime}, R_{-i}\right) \in X_{i \in N} \mathcal{R}_{i}$ such that $R_{i}^{\prime}$ is an $x$-reshuffling of $R_{i}$, then $f\left(R_{i}^{\prime}, R_{-i}\right) \in E\left(R_{i}, x\right)$.
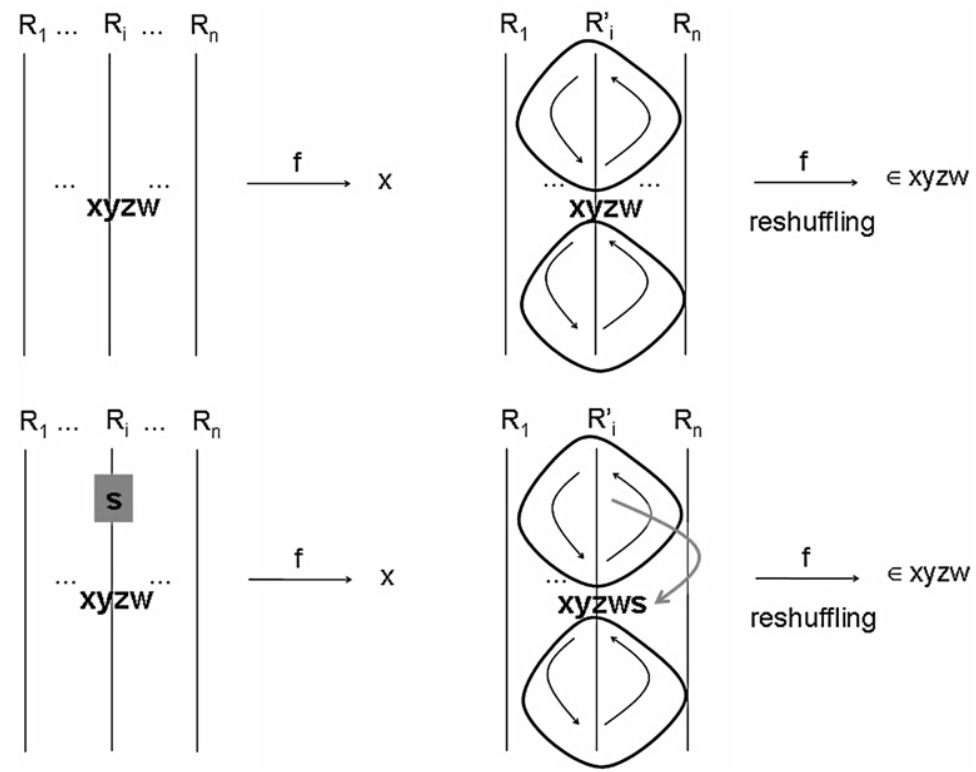

Fig. 2. Reshuffling invariance of $f$.

In words: If an alternative $x$ is chosen at a profile, some alternative in $x$ 's indifference class at this profile must be chosen at any other profile obtained from an $x$-reshuffling of agent $i$ 's preferences. Notice that in the case of strict preferences $x$ must be obtained at the new profile.

The following proposition marks the start of our research. We remark that monotonicity and reshuffling invariance, our two independent conditions, are necessary for any social choice function defined on any domain to be strategy-proof, and that they are, moreover, also sufficient for the universal domain. However, we also show that our conditions are not always sufficient to guarantee strategy-proofness: we present a rule satisfying both of them which is nevertheless manipulable. ${ }^{4}$

\section{Proposition 1.}

(1) Reshuffling invariance and monotonicity are necessary conditions for a social choice function to be strategy-proof, regardless of the cartesian domain on which $f$ is defined.

\footnotetext{
3 Note that given the definition, $x$-reshufflings of $R_{i}$ are also $y$-reshufflings of $R_{i}$, for any $y \in E\left(R_{i}, x\right)$. This is why, even if we are admitting indifference classes, the notion of a reshuffling around an alternative does not need any further qualifications.

4 Our two conditions factorize and separate two types of transformations that are sometimes mixed together in the same requirement. Even then, further factorizations are conceivable. For example, in Barberà and Dutta (1982) it becomes useful to distinguish between reshufflings that occur in the upper contour set of an alternative and those that involve its lower contour set only. But we stay with our simple conditions, which we find very intuitive and clear to relate to strategy-proofness.
} 
(2) For social choice functions defined on the universal domain of preferences over alternatives, reshuffling invariance and monotonicity are necessary and sufficient conditions for the strategy-proofness of $f$.

(3) There exist cartesian domains of preferences and social choice functions defined on them for which satisfying reshuffling invariance and monotonicity is not sufficient for $f$ to be strategy-proof.

Proof. To prove part (1) of Proposition 1, let $f$ be a strategy-proof social choice function defined on a cartesian domain $\times_{i \in N} \mathcal{R}_{i}$. Suppose first that $f$ violates reshuffling invariance, that is, there exist $R_{N}$ such that $f\left(R_{N}\right)=x$ and $R_{i}^{\prime}$ an $x$ reshuffling of $R_{i}$ such that $f\left(R_{i}^{\prime}, R_{-i}\right) \notin E\left(R_{i}, x\right)$. If either $f\left(R_{i}^{\prime}, R_{-i}\right) P_{i}^{\prime} x$ or else $f\left(R_{i}^{\prime}, R_{-i}\right) I_{i}^{\prime} x$ (the latter holds if $f\left(R_{i}^{\prime}, R_{-i}\right) \in$ $\left.E\left(R_{i}^{\prime}, x\right) \backslash E\left(R_{i}, x\right)\right)$ then $f\left(R_{i}^{\prime}, R_{-i}\right) P_{i} x=f\left(R_{N}\right)$ which is a contradiction to strategy-proofness. Otherwise, if $x P_{i}^{\prime} f\left(R_{i}^{\prime}, R_{-i}\right)$ then we have obviously a contradiction to strategy-proofness.

Suppose now that $f$ violates monotonicity, that is, there exist $R_{N}$ such that $f\left(R_{N}\right)=x$ and $R_{i}^{\prime}$ an $x$-monotonic transformation of $R_{i}$ such that $f\left(R_{i}^{\prime}, R_{-i}\right) \notin E\left(R_{i}, x\right)$. Thus, $f\left(R_{i}^{\prime}, R_{-i}\right) \notin B_{x}^{R_{i}}$. Observe that either $f\left(R_{i}^{\prime}, R_{-i}\right) P_{i} x$ or else $x P_{i} f\left(R_{i}^{\prime}, R_{-i}\right)$ holds. For the latter, observe that since $R_{i}^{\prime}$ is an $x$-monotonic transformation of $R_{i}, x P_{i}^{\prime} f\left(R_{i}^{\prime}, R_{-i}\right)$. Then, in both cases we obtain the corresponding contradictions to strategy-proofness.

The proof of part (2) is postponed, as it is a corollary of our general result in Theorem 1.

To prove part (3), an example with strict preferences will suffice. Consider a problem with three voters $N=\{1,2,3\}$ and two candidates $a$ and $b$, to be elected to join a club. The alternatives are to choose both, or only one of them, or none of the two. Hence, alternatives are sets of candidates $A=\{\emptyset, a, b,\{a, b\}\}$.

Given a preference on sets, candidates are called good if they are better than the empty set, when chosen alone, and bad otherwise. Preferences are separable if adding a good candidate to any set makes the union better, and adding a bad one makes the union worse. Our example refers to a voting rule defined on the domain of separable preferences for this case of two candidates, four alternatives and three voters. The set of individual separable preferences is:

\begin{tabular}{cccccccc}
\hline$R^{1}$ & $R^{2}$ & $R^{3}$ & $R^{4}$ & $R^{5}$ & $R^{6}$ & $R^{7}$ & $R^{8}$ \\
\hline$\emptyset$ & $\emptyset$ & $a$ & $a$ & $b$ & $b$ & $\{a, b\}$ & $\{a, b\}$ \\
$a$ & $b$ & $\emptyset$ & $\{a, b\}$ & $\emptyset$ & $\{a, b\}$ & $a$ & $b$ \\
$b$ & $a$ & $\{a, b\}$ & $\emptyset$ & $\{a, b\}$ & $\emptyset$ & $b$ & $a$ \\
$\{a, b\}$ & $\{a, b\}$ & $b$ & $b$ & $a$ & $a$ & $\emptyset$ & $\emptyset$ \\
\hline
\end{tabular}

Define the rule as the Borda count on $A$ with tie breaking. Voters rank the four alternatives, and each alternative gets three points whenever a voter ranks it first, two when ranked second, one when third and none if last. The choice is the alternative with the highest sum of points, if unique. As for possible ties, notice that, in our example, when there is a tie for first position. There may be at most one voter for whom none of the tied alternatives is the best for him. If there is such an individual, the tie is broken in favor of that alternative that he prefers. Otherwise, the tie is broken according to a pre-determined order of alternatives, say $O:\{a, b\}, b, a, \emptyset$.

Notice that the only cases where the antecedents of reshuffling invariance and monotonicity would apply are those where we change a preference to another having the same top. Given that, it is easy to see that both conditions are respected in our example.

Yet, observe that the function is still manipulable. To see that, let $R=\left(R^{1}, R^{6}, R^{7}\right), R^{\prime}=\left(R^{6}, R^{6}, R^{7}\right)$. Then, $f(R)=b$ ( $b$ and $\{a, b\}$ have the same score and agent 1 breaks the tie) and $f\left(R^{\prime}\right)=\{a, b\}(b$ and $\{a, b\}$ have the same score but all agents have $b$ or $\{a, b\}$ as best alternative, so we use 0 ). Thus, agent 1 manipulates $f$ at $R^{\prime}$ via $R^{1}$. This ends the proof of Proposition 1.

Proposition 1 sets the ground for our main research question. Given that our two conditions are necessary and sufficient for strategy-proofness in the universal domain, but not sufficient for other cases where the domain is restricted, can we tell apart those domains where sufficiency holds, from those where it does not? As we shall see, we can. Moreover, our research leads us to define a type of domains which are interesting of their own right, for reasons we will discuss along the paper.

\section{Intertwined domains}

We now introduce our notion of intertwined domains. Whether a domain is intertwined or not will turn out to be crucial to determine whether the different conditions we are interested in may or may not be equivalent, when applied to social choice functions defined on such domains.

Before we provide a formal definition, let us describe the condition informally for the case of strict preferences (see Fig. 3). Select any two (strict) preferences $R$ and $R^{\prime}$, and any two alternatives $x$ and $y$, where $x P y$ (the relationship between the two in $R^{\prime}$ can be any). Suppose that there exists in our domain a third preference $\bar{R}$ such that one can transform $R$ into $\bar{R}$, through a sequences of changes in the positions of alternatives, such that these changes, at each step, simply consist in lifting the position of $y$, or of reshufflings around $y$. Suppose that one can also transform $R^{\prime}$ into $\bar{R}$ through another 


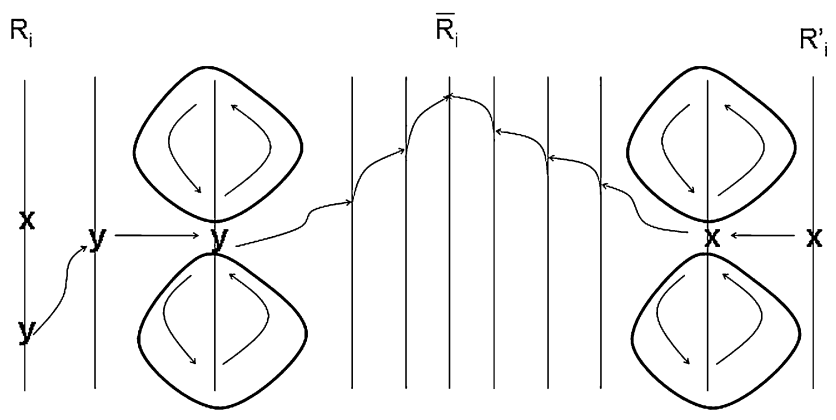

Fig. 3. Intertwined with strict preferences.

sequence of the same type of transformations, this time with liftings of $x$ and reshufflings around $x$. We will then say that $R$ and $R^{\prime}$ are $(x, y)$-intertwined. ${ }^{5}$

A domain of preferences will be intertwined if and only if any two of the preferences it contains are intertwined for any two alternatives.

Even more informally, we can say that an intertwined domain is one where one can travel from any pair of preferences to some intermediary preference just by lifting and reshuffling alternatives.

We now proceed to our formal definitions.

Definition 7. Let $R_{i} \in \mathcal{R}_{i}$ and $x \in A$. We say that $R_{i}^{\prime} \in \mathcal{R}_{i}$ is an $x$-pure reshuffling of $R_{i}$ if $(\mathrm{i}) \bar{L}\left(R_{i}, x\right)=\bar{L}\left(R_{i}^{\prime}, x\right)$, and (ii) $\bar{U}\left(R_{i}^{\prime}, x\right)=\bar{U}\left(R_{i}, x\right){ }^{6}$

Definition 8. Let $R_{i}, \bar{R}_{i} \in \mathcal{R}_{i}$ and $x \in A$. We say that $\bar{R}_{i}$ is an $x$-direct transform of $R_{i}$ if either $\bar{R}_{i}$ is an $x$-pure reshuffling of $R_{i}$ or $\bar{R}_{i}$ is an $x$-monotonic transformation of $R_{i}$.

In what follows, we use again the notation $B_{x}^{R_{t}}$ that was introduced when defining $x$-monotonic transformations (Definition 3). When specializing Definitions 9-12, to the case of strict preferences, the reader should keep in mind that then $B_{x}=x$.

Definition 9. Let $R_{i}, \bar{R}_{i} \in \mathcal{R}$ and $x \in A$. We say that $\bar{R}_{i}$ is an $x$-transform of $R_{i}$ if there exist a sequence of preferences $R_{1}, R_{2}, \ldots, R_{T}$ such that $R_{1}=R_{i}, R_{T}=\bar{R}_{i}$, and for any $t \in(1, T]$, each $R_{t}$ is an $x$-direct transform of $R_{t-1}$ where $B_{x}^{R_{t-1}}=B_{x}$ for each $t$ when the $x$-direct transform of $R_{t-1}$ is an $x$-monotonic transformation.

The set $B_{x}$ will consist of those alternatives (including $x$ itself) that are indifferent to $x$ in $R_{i}$ and that will be "lifted" along with $x$ in the sequence of intertwined leading from $R_{i}$ to $\bar{R}_{i}$. Our qualification is that, although $x$ may be accompanied by some of these indifferent alternatives along the sequence, those that are lifted once continue to be lifted all along.

Definition 10. Let $R_{i}, R_{i}^{\prime} \in \mathcal{R}_{i}, x, y \in A$ where $x P_{i} y$. We say that $R_{i}$ is $(x, y)$-intertwined with $R_{i}^{\prime}$ if there exists $\bar{R}_{i} \in \mathcal{R}_{i}$ such that $\bar{R}_{i}$ is both a $y$-transform of $R_{i}$ and an $x$-transform of $R_{i}^{\prime}$ where $B_{y} \subseteq E\left(R_{i}, y\right) \backslash\left[E\left(R_{i}^{\prime}, x\right) \backslash\{y\}\right]$ and $B_{x} \subseteq E\left(R_{i}^{\prime}, x\right) \backslash E\left(R_{i}, y\right)$.

As already remarked after Definition 9 , the sets $B_{x}$ and $B_{y}$ are those that accompany $x$ and $y$, respectively, in the monotone transformations leading from $R_{i}^{\prime}$ and from $R_{i}$ to $\bar{R}_{i}$. In the definition above we impose the additional requirement that these two sets do not intersect: if $E\left(R_{i}, y\right)$ and $E\left(R_{i}^{\prime}, x\right)$ contain some common elements, these can join either $y$ or $x$ in the monotone transformations from $R_{i}$ or from $R_{i}^{\prime}$, but not both of them.

Definition 11. A set of individual preferences $\mathcal{R}_{i}$ is intertwined if for any $R_{i} \in \mathcal{R}_{i}$, for any $x, y \in A$ such that $x P_{i} y$, and any $R_{i}^{\prime} \in \mathcal{R}_{i}, R_{i}$ is $(x, y)$-intertwined with $R_{i}^{\prime}$.

Definition 12. A domain $X_{i \in N} \mathcal{R}_{i}$ is intertwined if for any agent $i, \mathcal{R}_{i}$ is intertwined.

We are now ready to state our equivalence result.

\footnotetext{
${ }^{5}$ Notice that being $(x, y)$-intertwined is not the same as $(y, x)$-intertwined as emphasized in Definition 10 below.

${ }^{6}$ Note that a pure reshuffling as defined here is a particular case of reshufflings, as they appear in Definition 5. Both refer to transformations of preferences. But our definition here will be used for the purpose of restricting domains of preferences, while in the preceding section Definition 5 was later used to establish a condition on social choice functions. Their uses here and there are, in principle, logically independent.
} 
Theorem 1. Any social choice function defined on an intertwined domain is strategy-proof if and only if it satisfies monotonicity and reshuffling invariance.

Proof. That these conditions are necessary for strategy-proofness is already proven, as part (1) of Proposition 1 above. To prove that they are sufficient, let us proceed by contradiction. Let $\chi_{i \in N} \mathcal{R}_{i}$ be an intertwined domain and assume that $f$ satisfies reshuffling invariance and monotonicity, but is manipulable. That is, there exist $R_{N} \in \mathrm{X}_{i \in N} \mathcal{R}_{i}$ and $R_{i}^{\prime} \in \mathcal{R}_{i}$ such that $x=f\left(R_{i}^{\prime}, R_{-i}\right) P_{i} f\left(R_{N}\right)=y$.

Take $R_{i}, R_{i}^{\prime} \in \mathcal{R}_{i}, x, y \in A$ such that $x P_{i} y$. Then, by intertwinedness, $R_{i}$ is $(x, y)$-intertwined with $R_{i}^{\prime}$. That is, there exists $\bar{R}_{i} \in \mathcal{R}_{i}$ such that $\bar{R}_{i}$ is both a $y$-transform of $R_{i}$ and an $x$-transform of $R_{i}^{\prime}$ where $B_{y} \subseteq E\left(R_{i}, y\right) \backslash\left[E\left(R_{i}^{\prime}, x\right) \backslash\{y\}\right]$, $B_{x} \subseteq E\left(R_{i}^{\prime}, x\right) \backslash E\left(R_{i}, y\right)$. By definition, $x \in B_{x}$ and $y \in B_{y}$. We distinguish two cases.

Case 1. $E\left(R_{i}, y\right) \cap E\left(R_{i}^{\prime}, x\right)=\emptyset$.

By reshuffling invariance and monotonicity the following two conditions hold: on the one hand, $f\left(\bar{R}_{i}, R_{-i}\right) \in E\left(R_{i}, y\right)$ and on the other hand $f\left(\bar{R}_{i}, R_{-i}\right) \in E\left(R_{i}^{\prime}, x\right)$ which is the desired contradiction since $E\left(R_{i}, y\right) \cap E\left(R_{i}^{\prime}, x\right)=\emptyset$.

Case 2. $E\left(R_{i}, y\right) \cap E\left(R_{i}^{\prime}, x\right) \neq \emptyset .^{7}$

Observe first that if $\bar{R}_{i}=R_{i}^{\prime}$ then $f\left(R_{i}^{\prime}, R_{-i}\right) \in E\left(R_{i}, y\right)$ and since $x \notin E\left(R_{i}, y\right)$ then $f\left(R_{i}^{\prime}, R_{-i}\right) \neq x$ which is a contradiction. Thus, $\bar{R}_{i} \neq R_{i}^{\prime}$.

Note that in Case 2, neither only by $y$-pure reshufflings of $R_{i}$ and/or by $x$-pure reshufflings of $R_{i}^{\prime}$, nor by $x$-pure reshuffling of $R_{i}^{\prime}$ and $y$-monotonic transforms of $R_{i}$ can be enough to connect $R_{i}$ and $R_{i}^{\prime}$ in an intertwined way. In the latter case note that $B_{y}$ cannot join $x$ (it is not a feasible monotonic transformation). Thus, there must exist $R_{t}, R_{t+1}$ such that $R_{t+1}$ is an $x$-monotonic transformation of $R_{t}$. That is, $x$ must go up at some step. Without loss of generality, suppose that the first time $x$ goes up is for $t=1$. That is, $R_{1}=R_{i}^{\prime}$ and $R_{2}$ is an $x$-monotonic transformation of $R_{i}^{\prime}, x \in B_{x}$ by definition. By monotonicity of $f, f\left(R_{2}, R_{-i}\right) \in E\left(R_{i}^{\prime}, x\right)$.

Subcase 2.1. Suppose that $f\left(R_{2}, R_{-i}\right) \in B_{x}$.

By definition of intertwinedness, $B_{x} \cap E\left(R_{i}, y\right)=\emptyset$. Then, on the one hand, going from $R_{i}^{\prime}$ to $\bar{R}_{i}$ by consecutively applying monotonicity or reshuffling invariance, $f$ selects alternatives in $B_{x}$. On the other hand, going from $R_{i}$ to $\bar{R}_{i}$ by consecutively applying monotonicity or reshuffling invariance, $f$ selects alternatives in $E\left(R_{i}, y\right)$ which is a contradiction of $f$ being a function.

Subcase 2.2. Suppose $f\left(R_{2}, R_{-i}\right) \notin B_{x}$, thus $f\left(R_{2}, R_{-i}\right) \neq x$. Then we get a contradiction to the fact that $f$ satisfies reshuffling invariance (going from $R_{2}$ to $R_{i}^{\prime}, B_{x}$ goes down and joins $E\left(R_{i}^{\prime}, x\right) \backslash B_{x}$; that is, $R_{i}^{\prime}$ is an $x$-reshuffling of $R_{2}$. By reshuffling invariance, $f\left(R_{i}^{\prime}, R_{-i}\right) \in E\left(R_{i}^{\prime}, x\right) \backslash B_{x}$ which is false since $\left.f\left(R_{i}^{\prime}, R_{-i}\right)=x \in B_{x}\right)$.

This completes the proof.

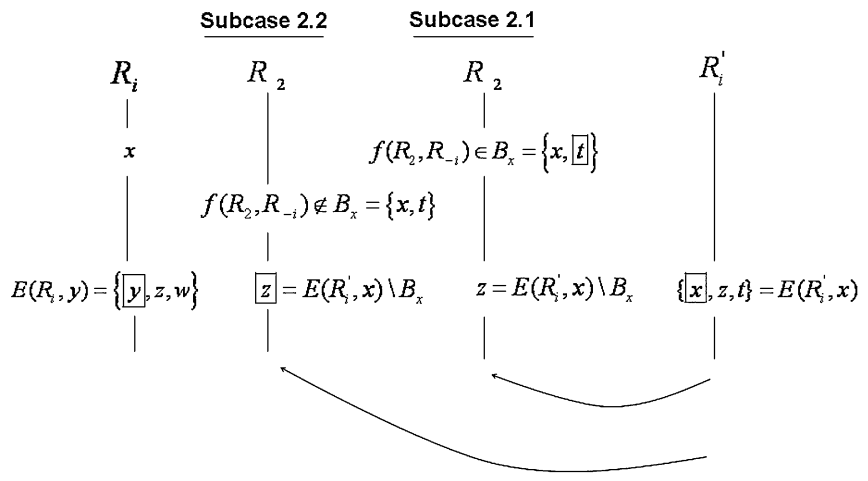

Fig. 4. An illustration of the argument in Case 2 for specific preferences $R_{i}, R_{i}^{\prime}$ and $R_{2}$. The chosen alternative is the boxed one.

${ }^{7}$ See Fig. 4 for a particular illustration that may help the reader to follow the arguments in the proof of Case 2. 
Note that if all agents have strict preferences, that is $\chi_{i \in N} \mathcal{P}_{i}$, the equivalence between strategy-proofness and our two conditions still holds. The same proof would work and only Case 1 would be relevant.

Therefore, being defined on an intertwined domain is sufficient to guarantee equivalence between our two properties and strategy-proofness. However, intertwinedness is not a necessary domain condition for this equivalence to hold. Example 1 presents a non-intertwined domain where every monotonic and reshuffling invariant social choice function is strategy-proof.

Example 1. Let $N=\{1,2\}, A=\{x, y, z, v\}$, and $\mathcal{P}_{1}=\mathcal{P}_{2}=\left\{R^{1}, R^{2}, R^{3}\right\}$ where:

\begin{tabular}{ccc}
\hline$R^{1}$ & $R^{2}$ & $R^{3}$ \\
\hline$v$ & $v$ & $x$ \\
$x$ & $x$ & $v$ \\
$y$ & $z$ & $z$ \\
$z$ & $y$ & $y$ \\
\hline
\end{tabular}

Note first that this domain is not intertwined: $R^{1}$ is not $x y$-intertwined with $R^{3}$.

By construction, one can also check that any social choice function satisfying monotonicity and reshuffling invariance is strategy-proof.

To check which monotonic and reshuffling invariant rules we can construct, consider the following four cases

Case 1: $f\left(R^{1}, R^{1}\right)=\chi$,

Case 2: $f\left(R^{1}, R^{1}\right)=z$,

Case 3: $f\left(R^{1}, R^{1}\right)=y$,

Case 4: $f\left(R^{1}, R^{1}\right)=v$.

If Case 1 or Case 2 holds, we obtain the constant rule $x$ or $z$, respectively. If Case 3 holds, we obtain either the constant rule $y$ or else 4 rules with binary range $y, z$. If Case 4 holds, we obtain either the constant rule $v$ or else 4 rules with binary range $v, x$. Thus, in total we may have 12 monotonic and reshuffling invariant rules. It is easy to check that all of them are strategy-proof.

We finish this section with two remarks for the very careful reader. First, note that the requirement " $x P_{i} y$ " in Definition 11 of an intertwined set of preferences bites. By ruling out that assumption, we would get a stronger domain condition, that would be violated by the set of strict single-peaked preferences in the problem of the provision of a public good and also by the set of all strict preferences (as shown in Example 2).

Example 2. Let $N=\{1,2\}, A=\{x, y, z\}$, and $\mathcal{P}_{1}=\mathcal{P}_{2}=\left\{R^{1}, R^{2}, R^{3}, R^{4}\right\}$ where:

\begin{tabular}{cccc}
\hline$R^{1}$ & $R^{2}$ & $R^{3}$ & $R^{4}$ \\
\hline$x$ & $y$ & $y$ & $z$ \\
$y$ & $x$ & $z$ & $y$ \\
$z$ & $z$ & $x$ & $x$ \\
\hline
\end{tabular}

$\mathcal{P}_{1}$ is the set of all single-peaked preferences (given the order $x<y<z$ ) which we later show that are intertwined. Observe that $x P^{1} y$ (i.e. $y$ is not strictly preferred to $x$ for $R^{1}$ ) and $R^{1}$ is not $(y, x)$ - "intertwined" with $R^{3}$ in the stronger sense.

Consider $\widetilde{\mathcal{P}}_{i}=\left\{R^{1}, R^{2}, R^{3}, R^{4}, R^{5}, R^{6}\right\}$ where $x P^{5} z P^{5} y$ and $z P^{6} x P^{6} y$. Note that $\widetilde{\mathcal{P}}_{i}$ is the set of all strict preferences on $A$. Adding $R^{5}$ and $R^{6}$ does not help to $(y, x)$-“intertwin" $R^{1}$ with $R^{3}$.

Second, observe that we could have considered a weaker version of reshuffling invariance, say WRI, imposing some condition only for pure reshufflings. That is, graphically WRI would be represented by the above part in Fig. 2 . In such case though, our intertwined domain condition is not sufficient to guarantee that WRI and monotonicity imply strategy-proofness (see Example 3).

Example 3. Let $N=\{1,2\}, A=\{x, y, z\}$, and $\mathcal{R}_{1}=\mathcal{R}_{2}=\left\{R^{1}, R^{2}, R^{3}\right\}$ where:

\begin{tabular}{ccc}
\hline$R^{1}$ & $R^{2}$ & $R^{3}$ \\
\hline$y$ & $y$ & $y$ \\
$z$ & $z x$ & $x$ \\
$x$ & & $z$
\end{tabular}

The reader can carefully check that $\mathcal{R}_{i}$ is intertwined. Let $f$ be such that $f\left(R^{1}, R_{2}\right)=f\left(R^{3}, R_{2}\right)=x, f\left(R^{2}, R_{2}\right)=z$ for any $R_{2} \in \mathcal{R}^{2}$. Observe that $f$ satisfies monotonicity (it only needs to be checked when agent 1 changes preferences from $R^{3}$ 
to $R^{1}$ ) and $f$ satisfies weak reshuffling invariance too (trivially, since there is no pure reshuffling at some alternative in the range). Clearly, $f$ is manipulable by agent 1: $f\left(R^{2}, R_{2}\right)=z P^{1} x=f\left(R^{1}, R_{2}\right)$. Observe also that $f$ violates reshuffling invariance ( $R^{2}$ is a reshuffling of $R^{1}$ at $x$, thus $f\left(R^{2}, R_{2}\right)$ should belong to $E\left(R^{1}, x\right)=x$ which is not the case).

The interested reader may also wonder whether a weaker notion of intertwinedness (one where we would not insist in pure reshufflings only) might still allow for an analogous equivalence to the one we get in Theorem 1 . We do not know. But we cannot find of relevant cases that would then be covered and that are not already accounted for under our present definition.

\section{Examples}

In this section, we show that several interesting preference domains are indeed intertwined. Some of the examples come from the classical social choice tradition, but we also present domains that involve the allocation of private goods. It is precisely because of these applications that we have insisted in preferences allowing for indifferences (since selfish agents are indifferent among the many allocations where they receive the same amount of goods), and also in domains where the families of admissible preferences for different agents are not the same (since agents value the same allocation by looking at their different components, which, in addition, may come from personalized sets of consequences).

In the case of pure public goods, domain restrictions can be expressed directly in terms of the alternatives involved. This is the case for the main domains that we start with. Note that the same proof of Propositions 2 and 3 below would work to state that the strict versions of the domains analyzed in each one of the propositions are also intertwined.

\subsection{Universal domain}

For individual preferences, the universal domain is the set of all complete, reflexive, and transitive binary relations on $A$; that is, the universal domain is $\mathcal{R}_{i}=\mathcal{R}$ (see Section 2).

Before showing intertwinedness when the set of individual preferences is the universal domain, consider the following fact, that applies to any set of individual preferences.

Fact 1. Any preferences $R_{i}, R_{i}^{\prime} \in \mathcal{R}$ that share the same set of best alternatives $T$ are $x$-pure reshufflings of each other for any $x \in T$.

Proposition 2. The universal domain is intertwined.

Proof. Let $R_{i}, R_{i}^{\prime} \in \mathcal{R}_{i}=\mathcal{R}, x, y \in A$ where $x P_{i} y$.

Let $\bar{R}_{i} \in \mathcal{R}$ be a $y$-pure reshuffling of $R_{i}$ such that $p\left(\bar{R}_{i}\right)=x$ (if $x$ is the best alternative according to $R_{i}$ this step would not be necessary). Let $\widetilde{R}_{i} \in \mathcal{R}$ be a monotonic transformation of $R_{i}^{\prime}$ at $x$ such that $p\left(\widetilde{R}_{i}\right)=x$ (if $x$ is the best alternative according to $R_{i}^{\prime}$ this step would not be necessary). Note that $\bar{R}_{i}$ is an $x$-pure reshuffling of $\widetilde{R}_{i}$ by Fact 1 . This completes the proof.

\subsection{The single-peaked domain}

Single-peakedness arises as a natural restriction on the preferences of agents facing many relevant problems: determining the level of a pure public good without transfers, locating a facility on a line, deciding on a tax level, choosing among candidates, among others.

Definition 13. An individual preference $R_{i} \in \mathcal{R}$ is single-peaked on $A$ relative to a linear order $>$ of the set of alternatives if and only if

(1) $R_{i}$ has a unique maximal element $p_{i}\left(R_{i}\right)$, called the peak of $i$, and

(2) for all $y, z \in A$

$$
\left[z<y \leqslant p_{i}\left(R_{i}\right) \text { or } z>y \geqslant p_{i}\left(R_{i}\right)\right] \rightarrow y P_{i} z .
$$

Denote by $\mathcal{S}_{>} \subsetneq \mathcal{R}$ the set of individual preferences consisting of all single-peaked preferences relative to $>$.

We show that the set of all single-peaked preferences defined on $A$ is intertwined. Before that, we present two other facts that apply for single-peaked preferences.

Fact 2. For any single-peaked preference $R_{i} \in \mathcal{S}_{>}$, any $x$ and $y \in \bar{U}\left(R_{i}, x\right)$, there exists $R_{i}^{\prime}$ which is also single-peaked and an $x$-pure reshuffling of $R_{i}$. 
Fact 3. For any single-peaked preference $R_{i} \in \mathcal{S}_{>}$with peak $p\left(R_{i}\right)$ and any $x$ which is contiguous to $p\left(R_{i}\right)$ in the order $>$, the $x$-monotonic transformation of $R_{i}$ for which $x$ becomes top is also single-peaked.

Proposition 3. For any order $>$, the set of individual preferences $\mathcal{S}_{>}$is intertwined.

Proof. Let $R_{i} \in \mathcal{S}_{>}$and $x, y \in A$ such that $x P_{i} y$. Take any $R_{i}^{\prime} \in \mathcal{S}_{>}$. Without loss of generality, let $x<y$ according to the order on $A$. We have to show that $R_{i}$ is $(x, y)$-intertwined with $R_{i}^{\prime}$. Consider the following cases:

Case 1. $x R_{i}^{\prime} y$ and $p\left(R_{i}^{\prime}\right) \in(x, y)$.

Consider first $\bar{R}_{i}$ a $y$-pure reshuffling of $R_{i}$ such that $p\left(\bar{R}_{i}\right)=x$. It exists since we have all single-peaked preferences. Now, let $\widetilde{R}_{i} \in \mathcal{S}_{>}$be an $x$-pure reshuffling of $R_{i}^{\prime}$ such that $p\left(\widetilde{R}_{i}\right)=x+1$ (observe that if $x+1=y$, we could not be in Case 1). By Fact 2, $\widetilde{R}_{i}$ exists. Now, consider $\overline{\bar{R}}_{i}$ be an $x$-monotonic transformation of $\widetilde{R}_{i}$ such that $p\left(\overline{\bar{R}}_{i}\right)=x$. By Fact $3, \overline{\bar{R}}_{i}$ is single-peaked. Note that $\bar{R}_{i}$ and $\overline{\bar{R}}_{i}$ have the same peak thus by Fact $1, \bar{R}_{i}$ is an $x$-pure reshuffling of $\overline{\bar{R}}_{i}$. Thus, $\bar{R}_{i} \in \mathcal{S}$ is both a $y$-(direct) transform of $R_{i}$ and an $x$-transform of $R_{i}^{\prime}$ and therefore $R_{i}$ is $(x, y)$-intertwined with $R_{i}^{\prime}$.

Case 2. $x P_{i}^{\prime} y$ and $p\left(R_{i}^{\prime}\right) \leqslant x$.

Consider first $\bar{R}_{i}$ a $y$-pure reshuffling of $R_{i}$ such that $p\left(\bar{R}_{i}\right)=x$. It exists since we have all single-peaked preferences. If $p\left(R_{i}^{\prime}\right)=x$, clearly $\bar{R}_{i}$ is both a $y$-transform of $R_{i}$ and an $x$-transform (an $x$-pure reshuffling) of $R_{i}^{\prime}$ and thus $R_{i}$ is $(x, y$ )intertwined with $R_{i}^{\prime}$. Otherwise, if $p\left(R_{i}^{\prime}\right) \neq x$, let $\widetilde{R}_{i} \in \mathcal{S}_{>}$be an $x$-pure reshuffling of $R_{i}^{\prime}$ such that $p\left(\widetilde{R}_{i}\right)=x-1$. By Fact 2 , $\widetilde{R}_{i}$ exists. Now, consider $\overline{\bar{R}}_{i}$ be an $x$-monotonic transformation of $\widetilde{R}_{i}$ such that $p\left(\overline{\bar{R}}_{i}\right)=x$. By Fact 3 , $\overline{\bar{R}}_{i}$ is single-peaked. Note that $\bar{R}_{i}$ and $\overline{\bar{R}}_{i}$ have the same peak thus by Fact $1, \bar{R}_{i}$ is an $x$-reshuffling of $\overline{\bar{R}}_{i}$. Therefore, $\bar{R}_{i}$ is both a $y$-(direct) transform of $R_{i}$ and an $x$-transform of $R_{i}^{\prime}$ and thus $R_{i}$ is $(x, y)$-intertwined with $R_{i}^{\prime}$.

Case 3. $y P_{i}^{\prime} x$.

The same proof as in Case 1 works.

This completes the proof.

\subsection{Intertwined domains with private goods}

When we work with private goods, more structure is added to the description of the alternatives, and this additional structure suggests a re-definition of domain restrictions. We look here at this structure and at its implications on the definition of domain restrictions for the case of one private good. With $n$ agents, an alternative $a$ is an $n$-tuple of values $\left(a_{1}, \ldots, a_{n}\right)$, one for each agent.

Let $A_{i}$ be the set of values that are admissible for the $i$-th component, and let $A \subseteq \prod_{i \in N} A_{i}$. The idea of selfishness is associated with the assumptions that (1) each agent $i$ has a well-defined preference ordering on $A_{i}$, and (2) agent $i$ 's ordering of any pair of alternatives $a, a^{\prime} \in A$ is only based on her ordering of their $i$-th components $a_{i}$ and $a_{i}^{\prime}$.

Hence, given a set of alternatives $A \subseteq \prod_{i \in N} A_{i}$ and a set of preferences $\widetilde{\mathcal{R}}_{i}$ on $A_{i}$ for each agent $i \in N$, we can define the associated set of selfish preferences $\mathcal{R}_{i}$ on $A$, as the family such that, for all $a, a^{\prime} \in A, a R_{i} a^{\prime} \Leftrightarrow a_{i} \widetilde{R}_{i} a_{i}^{\prime}{ }^{8}$

It is clear that, under selfish preferences, indifferences among alternatives are mandatory since for all $i$ and any $a, a^{\prime} \in A$ such that $a_{i}=a_{i}^{\prime}$, any admissible preference $R_{i} \in \mathcal{R}_{i}$ must rank these two alternatives as indifferent. For the same reason, the sets $\mathcal{R}_{i}$ of admissible preferences must necessarily be different for different $i$ 's, since the admissible values for each component may be different, and moreover, their indifference classes for different agents cannot coincide (not even when all sets $A_{i}$ are equal).

Nevertheless, the following lemma allows us to extend the scope of our results to private good economies, with selfish agents.

Lemma 1. If $\mathcal{R}_{i}$ is the set of selfish preferences for agent $i$ on $A \subseteq \prod_{i \in N} A_{i}$ associated to $\widetilde{\mathcal{R}}_{i}$, and $\widetilde{\mathcal{R}}_{i}$ is intertwined, then $\mathcal{R}_{i}$ is also intertwined.

\footnotetext{
${ }^{8}$ Alternatively, we could take the $R_{i}$ relations as primitive and interpret selfishness as the condition guaranteeing that one can properly define $\widetilde{R}_{i}$, for all $i$, satisfying the above relationship.
} 
Proof. Let $x, y \in A, R_{i}, R_{i}^{\prime} \in \mathcal{R}_{i}$ such that $x P_{i} y$. By definition of $R_{i}, R_{i}^{\prime} \in \mathcal{R}_{i}$ on $A$, let $\widetilde{R}_{i}, \widetilde{R}_{i}^{\prime} \in \widetilde{\mathcal{R}}_{i}$ the corresponding associate preferences over $A_{i}$. By intertwined of $\widetilde{\mathcal{R}}_{i}$, there exists $\widetilde{\bar{R}}_{i}$ such that $\widetilde{\bar{R}}_{i}$ is a $y$-transform of $\widetilde{R}_{i}$ and an $x$-transform of $\widetilde{R}_{i}^{\prime}$. Let $\bar{R}_{i} \in \mathcal{R}_{i}$ be the associate preference to $\widetilde{\bar{R}}_{i}$ over $A$. Observe that $\bar{R}_{i}$ is both a $y$-transform of $R_{i}$ and an $x$-transform of $R_{i}^{\prime}$.

Let us now see how Lemma 1 allows us to extend our previous results to other interesting cases. We will consider, in turn, the housing problem, one-to-one matching or the problem of task rationing. ${ }^{9}$ In all three cases, $A$ can be represented by an $n$-tuple of objects.

\subsubsection{Housing markets}

In the case of housing, each component indicates which house (if any) is assigned to the corresponding tenant. Therefore, all sets $A_{i}$ are the same (they contain the names of all houses and a symbol to denote that no house is received). As for the admissible preferences $\widetilde{\mathcal{R}}_{i}$, it is usually assumed that they are all possible orders on $A_{i}$. Hence, since $\widetilde{\mathcal{R}}_{i}$ is intertwined because it is the set of universal preferences on $A_{i}$, so, $\mathcal{R}_{i}$ is also intertwined. Notice, however, that $\mathcal{R}_{i}$ is no longer the universal set of preferences on $A$. For example, strict preferences are ruled out by selfishness.

\subsubsection{Task rationing}

In the case of task rationing, each component indicates what fraction of the task is assigned to each member of the team. ${ }^{10}$ Again the possible values of $A_{i}$ are the same for each agent. Preferences $\widetilde{\mathcal{R}}_{i}$ on these fractions are assumed to be single-peaked. Hence, since $\widetilde{\mathcal{R}}_{i}$ is intertwined, so is $\mathcal{R}_{i}$.

\subsubsection{One-to-one matching}

In the case of one-to-one matching, each component will indicate what mate is attributed to each participant in the market. Notice that in previous two cases, the same set of objects are possible at each component. This is not the case with two-sided markets, where the $A_{i}$ 's are different for each agent (as they include all potential mates on the other side of the market, plus herself or himself). In matching (as in housing), the domain $\widetilde{\mathcal{R}}_{i}$ is unrestricted and thus intertwined. Again by Lemma $1, \mathcal{R}_{i}$ on $A$ in one-to-one matching is intertwined. ${ }^{11}$

\subsection{Non-intertwined domains}

We finish this section by presenting some examples of preferences violating intertwinedness. We start with the domain of separable preferences. ${ }^{12}$ In Example 4 we show this for two candidates, however that argument can be generalized whatever the number of candidates is.

Example 4. (see example in the proof of Proposition 1) Let $N=\{1,2,3\}$, two candidates $a, b$ can be elected: $A=$ $\{\emptyset, a, b,\{a, b\}\}$. The set of individual separable preferences is:

\begin{tabular}{cccccccc}
\hline$R^{1}$ & $R^{2}$ & $R^{3}$ & $R^{4}$ & $R^{5}$ & $R^{6}$ & $R^{7}$ & $R^{8}$ \\
\hline$\emptyset$ & $\emptyset$ & $a$ & $a$ & $b$ & $b$ & $\{a, b\}$ & $\{a, b\}$ \\
$a$ & $b$ & $\emptyset$ & $\{a, b\}$ & $\emptyset$ & $\{a, b\}$ & $a$ & $b$ \\
$b$ & $a$ & $\{a, b\}$ & $\emptyset$ & $\{a, b\}$ & $\emptyset$ & $b$ & $a$ \\
$\{a, b\}$ & $\{a, b\}$ & $b$ & $b$ & $a$ & $a$ & $\emptyset$ & $\emptyset$ \\
\hline
\end{tabular}

Observe that $R^{1}$ is not $(\emptyset, a)$-intertwined with $R^{3}$, that is, there does not exist a separable preference $\bar{R}_{i}$ such that $\bar{R}_{i}$ is both an $a$-transform of $R^{1}$ and an $\emptyset$-transform of $R^{3}$. For $\bar{R}_{i}$ to exist, the following should hold: we should be able to go from $R^{1}$ to $\bar{R}_{i}$ by means of $a$-direct transforms. In particular, $R_{2}$ in the chain $\left\{R_{t}\right\}, t=1, \ldots, k$ where $R_{1}=R^{1}$ and $R_{k}=R^{3}$, should be either a reshuffling of $R^{1}$ at $a$ or else a monotonic transformation of $R^{1}$ at $a$. However, neither one nor the other exist.

\footnotetext{
9 Our framework considers preferences used, for example, in Sprumont (1991) and Barberà et al. (1997) for rationing; in Pápai (2000), Bogomolnaia et al. (2005), Alcalde-Unzu and Molis (2011), Jaramillo and Manjunath (2011) for house allocation. For an introduction to matching, see Roth and Sotomayor (1990).

10 For expositional reasons, and in order to avoid the case of infinite alternatives, assume that only a finite set of job proportions are admissible. For example, that people can only be attributed a fraction $k / T$ of the task, where $k$ ranges from 0 to $K$.

11 Observe also that a similar argument would work to show that the domain of strict preferences with an outside option used in Ehlers (2002a) is intertwined.

12 This establishes that intertwinedness has bite. It is also consistent with our finding in this section that reshuffling invariance and monotonicity are not sufficient for strategy-proofness in domains that are not intertwined, and our example in the proof of part (3) of Proposition 1, where a function satisfying both conditions in a domain of separable preferences is manipulable.
} 
Although the domain of single-peaked preferences in the problem of the provision of a single public good satisfies intertwinedness, we can easily check that the symmetric single-peaked domain violates it. Similarly, the domain of singledipped preferences is not intertwined.

Example 5. Consider only three alternatives ordered as $x<y<z$, and consider $\mathcal{S}_{M}$ the domain of symmetric single-peaked preferences on $A=\{x, y, z\}$. There are three preferences in $\mathcal{S}_{M}: R^{1}$ with peak at $x, y$ second and $z$ the worst, $R^{2}$ with peak at $y$ where $x$ and $z$ are indifferent, and $R^{3}$ with peak $z, y$ second and $x$ the worst. Note that $R^{1}$ and $R^{2}$ are not $(y, z)$-intertwined. The same argument would allow us to say that a subset $\mathcal{R}$ of the strict single-peaked domain such that $\mathcal{R}=\left\{R^{1}, R^{3}, \bar{R}^{2}\right\}$ where $y \bar{P}^{2} x \bar{P}^{2} z$ violates intertwinedness.

Example 6. Consider four alternatives ordered as $x<y<z<v$, and consider $\mathcal{D}$ the domain of single-dipped preferences on $A=\{x, y, z, v\}$. There are eight preferences in $\mathcal{D}$ :

\begin{tabular}{cccccccc}
\hline$R^{1}$ & $R^{2}$ & $R^{3}$ & $R^{4}$ & $R^{5}$ & $R^{6}$ & $R^{7}$ & $R^{8}$ \\
\hline$v$ & $x$ & $v$ & $v$ & $v$ & $x$ & $x$ & $x$ \\
$z$ & $v$ & $z$ & $x$ & $x$ & $v$ & $y$ & $y$ \\
$y$ & $z$ & $x$ & $z$ & $y$ & $y$ & $v$ & $z$ \\
$x$ & $y$ & $y$ & $y$ & $z$ & $z$ & $z$ & $v$ \\
\hline
\end{tabular}

Note that $R^{1}$ is not $(z, y)$-intertwined with $R^{8}$.

Notice also that in the same framework of the provision of a single public good, the domains of single-plateaued, of weakly single-plateaued, and of weakly single-peaked preferences violates intertwinedness ("weakly" denoting that we allow indifference sets outside the top to be two closed intervals, one in each side of the plateau or the peak, respectively). However, each one of these domains of preferences satisfies a weaker domain condition for which our equivalence in Theorem 1 can still be established, with the same proof. Informally, the weaker version would consist in allowing that, in each step of the preferences path, subsequent subsets of the original $B_{y}$, each one containing $y$ (or $B_{x}$ containing $x$, respectively) were used to reshuffle or to push them up, instead of having $B_{y}$ fixed (a formal definition is available upon request).

We leave to the careful reader to check that other domains of preferences also fail to be intertwined. For example, the preferences used in Cantala (2004) when choosing a level of public good where agents' preferences are single-peaked but having an outside option, or in Miyagawa (2001), Barberà and Beviá (2002), and Ehlers (2002b) where the location of two (or more) public facilities is analyzed, are not intertwined domains.

\section{Further properties of social choice functions and their connections}

Until now we have explored the connection between strategy-proofness and the two properties of monotonicity and reshuffling invariance. This is because we find both of them very intuitive and because we think that factoring out the different requirements that can lead to strategy-proofness helps our understanding of what is crucial in order to achieve such a desirable property. Notice that rules satisfying monotonicity are abundant, and include the whole family of point voting rules. Hence, our decomposition helps to point at reshuffling invariance as the main culprit of the difficulties in designing strategy-proof rules.

At any rate, other authors have displayed alternative conditions which are also tightly connected with the notion of strategy-proofness. In this section, we discuss two of them, both defined for strict preferences. One is called strong monotonicity, and was proposed by Moulin (1988). The other is called strong positive association (see Muller and Satterthwaite, 1977). These are more synthetic properties, that combine in one single condition the type of responses to preference change that we have factored out in our two conditions. Throughout this section, we consider strict preferences to be able to compare our conditions with these two properties used in the literature.

Again, it is clear that strong positive association and strong monotonicity are necessary for strategy-proofness. Moreover, they are also sufficient, and thus equivalent to our conditions and to strategy-proofness itself, for functions defined on the universal domain. We show that these equivalences break down as we depart form this strong domain requirement and consider rules defined on smaller domains. We provide examples and results that clarify the eventual interdependence or independence of these different conditions. Yet, we also provide a result that re-enforces our previous findings about intertwined domains: for functions defined on any such domains (of which the universal one is a special case), the conjunction of monotonicity and reshuffling invariance is equivalent to strong monotonicity and also to strong positive association, with any of them being necessary and sufficient for strategy-proofness. This proves that, while different in a general setting, all of the intuitions expressed through these different properties are properly captured and become the same when rules are defined in intertwined domains, while not necessarily otherwise.

Before defining the conditions under discussion, for sake of comparison we write down the monotonicity condition for the case when agents' preferences are strict. 
Definition 14 (Definition 3 for strict preferences). A social choice function $f$ satisfies monotonicity on $X_{i \in N} \mathcal{P}_{i}$ if and only if for any $R_{N} \in \mathrm{X}_{i \in N} \mathcal{P}_{i}$ such that $f\left(R_{N}\right)=x$, and for any $R_{N}^{\prime} \in \mathrm{X}_{i \in N} \mathcal{P}_{i}$ satisfying the following conditions

(i) for any $i \in N$, for any $y \in A \backslash\{x\} ;\left[x P_{i} y \Rightarrow x P_{i}^{\prime} y\right]$, and

(ii) for any $i \in N$, for any $y, z \in A \backslash\{x\} ;\left[y P_{i} z \Leftrightarrow y P_{i}^{\prime} z\right]$.

Then, $f\left(R_{N}^{\prime}\right)=x$.

Definition 15. A social choice function $f$ satisfies strong positive association on $\chi_{i \in N} \mathcal{P}_{i}$ if and only if for any $R_{N}, R_{N}^{\prime} \in$ $\chi_{i \in N} \mathcal{P}_{i}$ such that for any agent $i \in N$ and any alternative $y \in A \backslash\left\{f\left(R_{N}\right)\right\},\left[f\left(R_{N}\right) P_{i} y \Rightarrow f\left(R_{N}\right) P_{i}^{\prime} y\right] ;$ then, $f\left(R_{N}^{\prime}\right)=f\left(R_{N}\right)$.

The difference between monotonicity and strong positive association is that in the latter we require invariance of the rule only for profiles satisfying part (i) of monotonicity as in Definition 14.

The other concept is strong monotonicity.

Definition 16. (See Definition 10.1 in Moulin, 1988.) A social choice function $f$ satisfies strong monotonicity on $X_{i \in N} \mathcal{P}_{i}$ if and only if for any $R_{N}, R_{N}^{\prime} \in \mathrm{X}_{i \in N} \mathcal{P}_{i}, R_{N} \neq R_{N}^{\prime}$ and $x \in A$, such that the following conditions hold:

(i) for any $i \in N$, for any $y \in A \backslash\{x\} ;\left[x P_{i} y \Rightarrow x P_{i}^{\prime} y\right]$, and

(ii) for any $i \in N$, for any $y, z \in A \backslash\{x\} ;\left[y P_{i} z \Leftrightarrow y P_{i}^{\prime} z\right]$.

Then, either $f\left(R_{N}^{\prime}\right)=f\left(R_{N}\right)$ or else $f\left(R_{N}^{\prime}\right)=x$.

Strong monotonicity implies our monotonicity condition, however that the converse is false (see Example 8 below).

Our first result summarizes the connections between properties in the general case.

Proposition 4. Let $f$ be a social choice social function defined on $\chi_{i \in N} \mathcal{P}_{i}$. Then, the following statements hold:

(1) If $f$ is strategy-proof then $f$ satisfies strong positive association.

(2) If $f$ satisfies strong positive association then $f$ satisfies reshuffling invariance and monotonicity.

(3) If $f$ satisfies strong positive association then $f$ satisfies strong monotonicity.

(4) If $f$ is strategy-proof then $f$ satisfies reshuffling invariance and monotonicity.

(5) If $f$ is strategy-proof then $f$ satisfies strong monotonicity.

Next we give four examples to show why some implications do not hold.

Example 7. Let $N=\{1,2\}, A=\{x, y, z, t\}$, and $\mathcal{P}_{1}=\mathcal{P}_{2}=\left\{R^{1}, R^{2}, R^{3}\right\}$ where:

\begin{tabular}{ccc}
\hline$R^{1}$ & $R^{2}$ & $R^{3}$ \\
\hline$x$ & $y$ & $y$ \\
$y$ & $x$ & $z$ \\
$y$ & $z$ & $x$ \\
\cline { 1 - 1 }$z$ & $t$ & $t$
\end{tabular}

Let $f$ be such that $f\left(R_{1}^{1}, R^{k}\right)=t$ and $f\left(R_{1}^{2}, R^{k}\right)=f\left(R_{1}^{3}, R^{k}\right)=y$, for any $R^{k} \in \mathcal{P}_{2}$. Note that $f$ satisfies strong positive association but $f$ is manipulable (by agent 1 at $\left(R_{1}^{1}, R^{k}\right)$ via $\left.R_{1}^{2}\right)$.

Example 8. Let $N=\{1,2\}, A=\{x, y, z\}$, and $\mathcal{P}_{1}=\mathcal{P}_{2}=\left\{R^{1}, R^{2}, R^{3}\right\}$ where:

\begin{tabular}{ccc}
\hline$R^{1}$ & $R^{2}$ & $R^{3}$ \\
\hline \hline$x$ & $y$ & $z$ \\
$y$ & $x$ & $y$ \\
\cline { 1 - 2 }$z$ & $z$ & $x$ \\
\hline
\end{tabular}

Observe that $\mathcal{P}_{1}$ is a subset of single-peaked preferences (given the order $x<y<z$ ). Define $f$ such that 1 is decisive as follows: $f\left(R_{1}^{3}, R_{2}\right)=y$ for any $R_{2} \in \mathcal{P}_{2}$, and $f\left(R_{1}, R_{2}\right)=x$, otherwise. We can show that $f$ satisfies reshuffling invariance 
and monotonicity but $f$ violates strong positive association $\left(f\left(R_{1}^{3}, R_{2}\right)=y \neq f\left(R_{1}^{2}, R_{2}\right)\right.$, while $\left.L\left(R_{1}^{3}, y\right) \varsubsetneqq L\left(R^{2}, y\right)\right)$, strategyproofness (agent 1 can manipulate $f$ at $\left(R_{1}^{2}, R_{2}\right)$ via $R_{1}^{3}$ ), and also strong monotonicity (observe that $R^{3}$ is a monotonic transformation of $R^{2}$ at $z$ according to conditions (i) and (ii) in strong monotonicity, however $f\left(R_{1}^{3}, R_{2}\right)=y \neq f\left(R_{1}^{2}, R_{2}\right)=x$ and $\left.f\left(R_{1}^{3}, R_{2}\right) \neq z\right)$.

Example 9. Let $A=\{x, y, z, t, l\}$, and for any $i \in N, \mathcal{P}_{i}=\left\{R^{1}, R^{2}, R^{3}, R^{4}\right\}$ :

\begin{tabular}{cccc}
\hline$R^{1}$ & $R^{2}$ & $R^{3}$ & $R^{4}$ \\
\hline$l$ & $z$ & $x$ & $l$ \\
$x$ & $l$ & $l$ & $x$ \\
$z$ & $y$ & $y$ & $y$ \\
$y$ & $x$ & $t$ & $z$ \\
\cline { 3 - 4 }$t$ & $t$ & $z$ & $t$ \\
\hline
\end{tabular}

Let $f$ be such that $f\left(R_{1}^{1}, \cdot\right)=f\left(R_{1}^{4}, \cdot\right)=t$ and $f\left(R_{1}^{2}, \cdot\right)=f\left(R_{1}^{3}, \cdot\right)=y$, for any $R_{-1} \in \mathcal{P}_{-1}$. Note that $f$ satisfies strong monotonicity but it violates both strong positive association and reshuffling invariance. Observe that $f\left(R_{1}^{4}, \cdot\right)=t \neq f\left(R_{1}^{3}, \cdot\right)=$ $y$ where $R^{3}$ is a reshuffling of $R^{4}$ at $f\left(R_{1}^{3}, \cdot\right)=y$, thus $f$ violates reshuffling invariance. To show that $f$ violates strong positive association, observe that $L\left(R^{3}, f\left(R_{1}^{3}, \cdot\right)\right) \supsetneqq L\left(R^{4}, f\left(R_{1}^{3}, \cdot\right)\right)$ but $f\left(R_{1}^{4}, \cdot\right)=t \neq f\left(R_{1}^{3}, \cdot\right)=y$.

Example 10. Let $A=\{x, y, z, w\}$ and the set of preferences of each agent is the following subset of single-peaked preferences on $A$ with respect to $x<y<w<z$ :

\begin{tabular}{cccc}
\hline$R^{1}$ & $R^{2}$ & $R^{3}$ & $R^{4}$ \\
\hline$x$ & $y$ & $w$ & $z$ \\
$y$ & $w$ & $y$ & $w$ \\
$w$ & $x$ & $z$ & $y$ \\
$z$ & $z$ & $x$ & $x$ \\
\hline
\end{tabular}

Let $p\left(R_{i}\right)$ be the best alternative in $A$ according to preference $R_{i}$. Define $f$ such that agent 1 is decisive as follows: $f\left(R_{1}, R_{-1}\right)=p\left(R_{1}\right)$, for any $R_{1} \neq R^{3}$ and for any $R_{-1} \in X_{j \in N \backslash\{1\}} \mathcal{P}_{j}$, and $f\left(R_{1}^{3}, R_{-1}\right)=z$ for any $R_{-1} \in X_{j \in N \backslash\{1\}} \mathcal{P}_{j}$. One can check that $f$ satisfies strong monotonicity but $f$ is manipulable (agent 1 would manipulate $f$ at $\left(R_{1}^{3}, R_{-1}\right)$ via $R_{1}^{2}$ ).

The proof of Proposition 4 consists, in fact, of the proof of part (3). The reason is that the proof of part (1) was already observed in Muller and Satterthwaite (1977) (when showing the same implication for the unrestricted strict domain, $\mathcal{P}^{n}$ ); the statement in part (2) is straightforward by definition; and parts (4) and (5) are obtained combining part (1) with (2) and (3), respectively.

Proof of part (3) of Proposition 4. Let $f$ be a social choice social function defined on $X_{i \in N} \mathcal{P}_{i}$ satisfying strong positive association. To show that $f$ satisfies strong monotonicity, take $R_{N}, R_{N}^{\prime} \in X_{i \in N} \mathcal{P}_{i}, R_{i}^{\prime} \neq R_{i}$ for some $i \in N$, and $x \in A$ such that conditions (i) and (ii) in the definition of strong monotonicity hold, that is,

(i) for any $i \in N$, for any $y \in A \backslash\{x\} ;\left[x P_{i} y \Rightarrow x P_{i}^{\prime} y\right]$, and

(ii) for any $i \in N$, for any $y, z \in A \backslash\{x\}$; $\left[y P_{i} z \Leftrightarrow y P_{i}^{\prime} z\right]$.

Suppose first that $f\left(R_{N}\right)=x$. Then, $f\left(R_{N}^{\prime}\right)=f\left(R_{N}\right)$ by strong positive association which ends the proof.

Suppose now that $f\left(R_{N}\right) \neq x$.

Without loss of generality, consider the order of agents $1,2, \ldots, n$ and change one by one individual preferences from $R_{i}$ to $R_{i}^{\prime}$, starting with agent 1 . That is,

Step 1. First change $R_{1}$ to $R_{1}^{\prime}$.

Case 1. Suppose that $x \in \bar{U}\left(R_{1}, f\left(R_{N}\right)\right)$. Then, $L\left(R_{1}, f\left(R_{N}\right)\right)=L\left(R_{1}^{\prime}, f\left(R_{N}\right)\right)$ and thus $f\left(R_{1}^{\prime}, R_{-1}\right)=f\left(R_{N}\right)$ by strong positive association.

Then, start again the argument of Step 1 changing $R_{2}$ by $R_{2}^{\prime}$ and replacing $R_{N}$ by $\left(R_{1}^{\prime}, R_{-1}\right)$ and $\left(R_{1}^{\prime}, R_{-1}\right)$ by $\left(R_{1}^{\prime}, R_{2}^{\prime}, R_{-1}\right)$. 
Case 2. Suppose that $x \in \bar{L}\left(R_{1}, f\left(R_{N}\right)\right)$. Then, either $x \in L\left(R_{1}, f\left(R_{N}\right)\right)=L\left(R_{1}^{\prime}, f\left(R_{N}\right)\right)$ or else $L\left(R_{1}^{\prime}, f\left(R_{N}\right)\right)=$ $L\left(R_{1}, f\left(R_{N}\right)\right) \backslash\{x\}$.

(2.a) If the former holds, $f\left(R_{1}^{\prime}, R_{-1}\right)=f\left(R_{N}\right)$ by strong positive association. Then, start again the argument of Step 1 changing $R_{2}$ by $R_{2}^{\prime}$ and replacing $R_{N}$ by $\left(R_{1}^{\prime}, R_{-1}\right)$ and $\left(R_{1}^{\prime}, R_{-1}\right)$ by $\left(R_{1}^{\prime}, R_{2}^{\prime}, R_{-1}\right)$.

(2.b) If $L\left(R_{1}^{\prime}, f\left(R_{N}\right)\right)=L\left(R_{1}, f\left(R_{N}\right)\right) \backslash\{x\}$ holds, consider two cases:

(2.b.1) If $f\left(R_{1}^{\prime}, R_{-1}\right) \in L\left(R_{1}^{\prime}, f\left(R_{N}\right)\right)=L\left(R_{1}, f\left(R_{N}\right)\right) \backslash\{x\}$ then $f\left(R_{1}^{\prime}, R_{-1}\right)=f\left(R_{N}\right)$ by strong positive association. Then, start again the argument of Step 1 changing $R_{2}$ by $R_{2}^{\prime}$ and replacing $R_{N}$ by $\left(R_{1}^{\prime}, R_{-1}\right)$ and $\left(R_{1}^{\prime}, R_{-1}\right)$ by $\left(R_{1}^{\prime}, R_{2}^{\prime}, R_{-1}\right)$.

(2.b.2) If $f\left(R_{1}^{\prime}, R_{-1}\right) \in \bar{U}\left(R_{1}^{\prime}, f\left(R_{N}\right)\right) \equiv \bar{U}\left(R_{1}, f\left(R_{N}\right)\right) \cup\{x\}$, we distinguish three subcases:

(2.b.2.1) If $f\left(R_{1}^{\prime}, R_{-1}\right) \in \bar{U}\left(R_{1}^{\prime}, x\right)$ : since $L\left(R_{1}, f\left(R_{1}^{\prime}, R_{-1}\right)\right)=L\left(R_{1}^{\prime}, f\left(R_{1}^{\prime}, R_{-1}\right)\right)$ we obtain that $f\left(R_{N}\right)=$ $f\left(R_{1}^{\prime}, R_{-1}\right)$ by strong positive association. Then, replace $R_{2}$ by $R_{2}^{\prime}$ and start again the argument of Step 1 replacing $R_{N}$ by $\left(R_{1}^{\prime}, R_{-1}\right)$ and $\left(R_{1}^{\prime}, R_{-1}\right)$ by $\left(R_{1}^{\prime}, R_{2}^{\prime}, R_{-1}\right)$.

(2.b.2.2) If $f\left(R_{1}^{\prime}, R_{-1}\right) \in \bar{U}\left(R_{1}^{\prime}, f\left(R_{N}\right)\right) \cap \bar{L}\left(R_{1}^{\prime}, x\right)$ : doing the same argument as in Case 2 we obtain that $f\left(R_{1}^{\prime}, R_{-1}\right)=f\left(R_{N}\right)$ by strong positive association.

Then, start again the argument of Step 1 changing $R_{2}$ by $R_{2}^{\prime}$ and replacing $R_{N}$ by $\left(R_{1}^{\prime}, R_{-1}\right)$ and $\left(R_{1}^{\prime}, R_{-1}\right)$ by $\left(R_{1}^{\prime}, R_{2}^{\prime}, R_{-1}\right)$.

(2.b.2.3) Let $f\left(R_{1}^{\prime}, R_{-1}\right)=x$. Then, start again the argument in Step 1 changing $R_{2}$ by $R_{2}^{\prime}$ and replacing $R_{N}$ by $\left(R_{1}^{\prime}, R_{-1}\right)$ and $\left(R_{1}^{\prime}, R_{-1}\right)$ by $\left(R_{1}^{\prime}, R_{2}^{\prime}, R_{-1}\right)$.

Repeating the same argument changing the preference of any agent we will obtain that either $f\left(R_{N}\right)=f\left(R_{N}^{\prime}\right)$ or else $f\left(R_{N}\right)=x$ which completes the proof.

In spite of this potential lack of equivalence, the fact is that all conditions become equivalent when applied to social choice functions defined in the strict universal domain (see Fig. 5 below). The equivalence between each of these conditions and strategy-proofness was indeed the starting point for different proofs of the Gibbard-Satterthwaite theorem. Rather than an isolated case, this equivalence extends to functions defined on any intertwined domain, as expressed in the following result.

Proposition 5. Let $f$ be a social choice function defined on an intertwined domain $\times_{i \in N} \mathcal{P}_{i}$. Then, the following statements are equivalent:

(i) $f$ satisfies reshuffling invariance and monotonicity.

(ii) $f$ is strategy-proof.

(iii) $f$ satisfies strong positive association.

The proof is a corollary of Theorem 1 and parts (1) and (2) of Proposition 4.

We can also obtain the following relationship.

Corollary 1. Let $X_{i \in N} \mathcal{P}_{i}$ be an intertwined domain. Then, any reshuffling invariant and monotonic rule satisfies also strong monotonicity.

The proof is straightforward by Theorem 1 and part (5) of Proposition 4. The following example shows that the converse of Corollary 1 does not hold.

Example 11. Let $N$ be a finite set of agents and $A=\{x, y, v, w, z\}$ be the set of alternatives. The set of admissible preferences is $\mathcal{P}=\left\{R^{1}, R^{2}, R^{3}, R^{4}, R^{5}, R^{6}\right\}$ where:

\begin{tabular}{|c|c|c|c|c|c|}
\hline$R^{1}$ & $R^{2}$ & $R^{3}$ & $R^{4}$ & $R^{5}$ & $R^{6}$ \\
\hline$x$ & $y$ & $y$ & $y$ & $y$ & $x$ \\
\hline$y$ & $x$ & $x$ & $x$ & $x$ & $y$ \\
\hline$v$ & $v$ & $v$ & $z$ & $z$ & $z$ \\
\hline$w$ & $w$ & $z$ & $v$ & $w$ & $w$ \\
\hline$z$ & $z$ & $w$ & $w$ & $v$ & $v$ \\
\hline
\end{tabular}




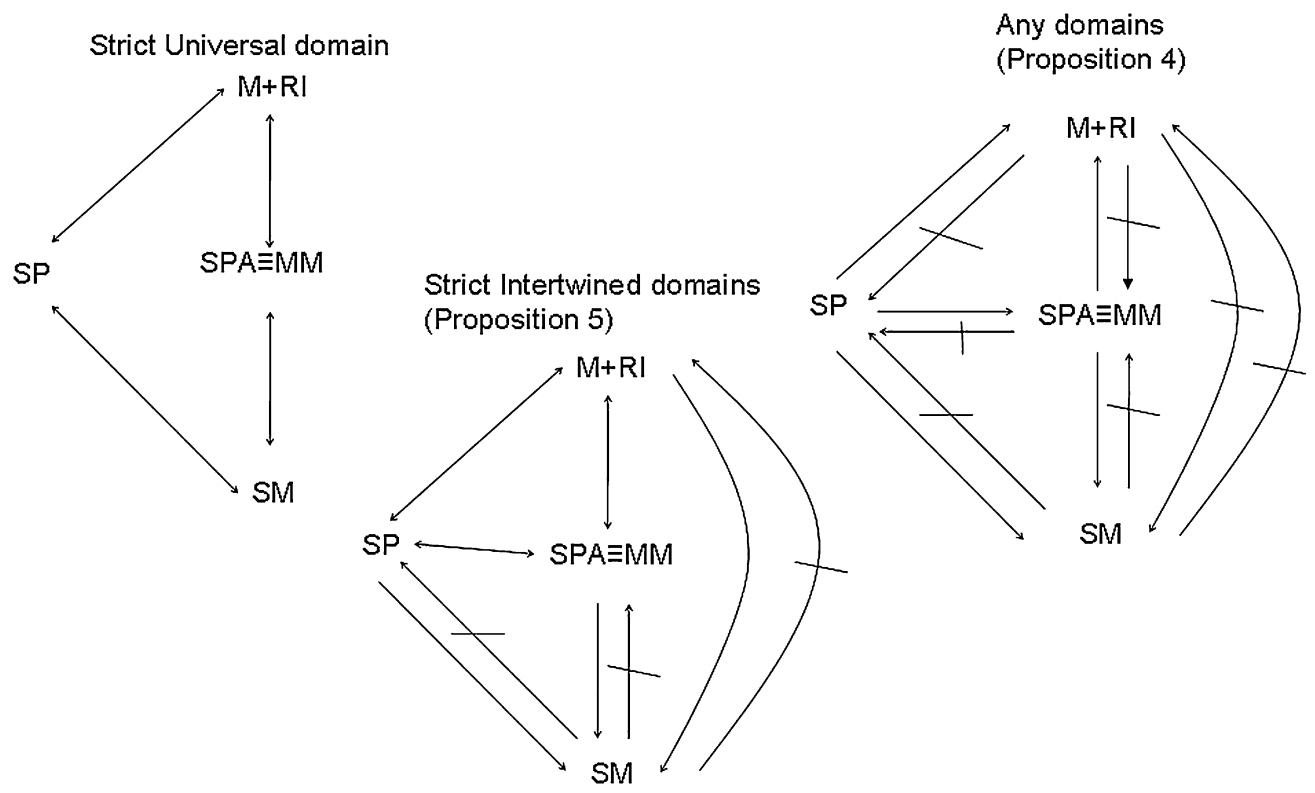

Fig. 5. Relationship between all properties. Note: $M=$ monotonicity, $M M=$ Maskin monotonicity, RI = reshuffling invariance, SM = strong monotonicity, $\mathrm{SP}=$ strategy-proofness, SPA = strong positive association.

We can check that $\mathcal{P}$ is an intertwined domain. ${ }^{13}$ Let $f$ be such that $f\left(R_{1}^{1}, R_{-1}\right)=x$ for any $R_{-1}$ and $f\left(R_{1}^{l}, R_{-1}\right)=y$ for $l=2,3,4,5,6$ and for any $R_{-1}$. Observe that $f$ satisfies strong monotonicity, thus monotonicity, however $f$ violates reshuffling invariance (since $R^{6}$ is a reshuffling of $R^{1}$ at $x, f\left(R_{1}^{6}, R_{-1}\right)$ should coincide with $f\left(R_{1}^{1}, R_{-1}\right)=x$ but it is not the case) and of course $f$ is manipulable (agent 1 would manipulate $f$ at $\left(R_{1}^{6}, R_{-1}\right)$ via $\left.R_{1}^{1}\right)$. To show that $f$ satisfies strong monotonicity, observe first that the only relevant comparisons are between $R_{1}^{1}$ and $R_{1}^{t}$ for $t=\{2,3,4,5,6\}$ since the outcome of $f$ differs. Comparing $R_{1}^{1}$ with $R_{1}^{t}$, for $t=\{3,4,5,6\}$ there is no direct monotonic transform (two pairs of alternatives are ordered differently in $R_{1}^{1}$ compared to $R_{1}^{t}$ ). For $R_{1}^{1}$ and $R_{1}^{2}$, $x$ overtakes $y$ or vice versa and strong monotonicity is satisfied.

In Fig. 5 we summarize all the general relationships stated in Proposition 4, the fact that the converse relationships do not hold in general, and also that reshuffling invariance and monotonicity, jointly, are neither necessary nor sufficient for strong monotonicity. Additionally, and for strict intertwined domains (that is, for intertwined domains with only strict preferences), we summarize the relationships stated in Proposition 5 and Corollary 1 and the fact that strong monotonicity does not imply the other properties.

\section{Intertwinedness and indirect sequential inclusion}

In this section and for the case of strict preferences we study the relationship between intertwinedness and another domain condition called indirect sequential inclusion. The latter was shown to be a sufficient condition to guarantee the equivalence between strategy-proofness and group strategy-proofness (see Barberà et al., 2010).

Obviously there are other domain conditions that could be analyzed since they turned out to be crucial to state interesting results in the social choice literature. However, the aim of the paper is not to provide an exhaustive comparison with all domains restrictions that have been proposed in the literature. For the domain we analyze in this section we are able to state additional implications with intertwinedness. We introduce two other domains restrictions in Appendix A that are independent with intertwinedness. ${ }^{14}$

Though (indirect) sequential inclusion is also defined with indifferences, we analyze only the case with strict preferences. To define (indirect) sequential inclusion, let $R_{N} \in X_{i \in N} \mathcal{R}_{i}$, and $y, z$ be a pair of alternatives and denote by $S\left(R_{N} ; y, z\right) \equiv$ $\left\{i \in N: y P_{i} z\right\}$ the set of agents who strictly prefer $y$ to $z$ according to their individual preferences in $R_{N}$.

\footnotetext{
13 The proof is case by case and it is available upon request.

14 Other interesting cases include circular domains (Sato, 2010a), domains satisfying Property T (Chatterji and Sen, 2011), and connected domains satisfying the non-restoration property (Sato, 2010b). Each one of these restrictions is also independent from that of intertwinedness (Examples 1 and 11 in this paper show non-equivalence for the later condition. Other examples are available upon request).
} 
Definition 17. Given a preference profile $R_{N} \in X_{i \in N} \mathcal{R}_{i}$ and a pair of alternatives $y, z \in A$, we define a binary relation $\succsim\left(R_{N} ; y, z\right)$ on $S\left(R_{N} ; y, z\right)$ as follows ${ }^{15}$ :

$$
i \succsim\left(R_{N} ; y, z\right) j \quad \text { if } L\left(R_{i}, z\right) \subseteq \bar{L}\left(R_{j}, y\right) .
$$

Note that the binary relation $\succsim$ must be reflexive but not necessarily complete. As usual, we can define the strict and the indifference binary relations associated to $\succsim$. Formally, $i \sim j$ if $L\left(R_{i}, z\right) \subseteq \bar{L}\left(R_{j}, y\right)$ and $L\left(R_{j}, z\right) \subseteq \bar{L}\left(R_{i}, y\right)$. We say that $i \succ j$ if $L\left(R_{i}, z\right) \subseteq \bar{L}\left(R_{j}, y\right)$ and $\neg\left[L\left(R_{j}, z\right) \subseteq \bar{L}\left(R_{i}, y\right)\right]$.

Definition 18. A preference profile $R_{N} \in X_{i \in N} \mathcal{R}_{i}$ satisfies sequential inclusion for $y, z \in A$ if the binary relation $\succsim\left(R_{N} ; y, z\right)$ on $S\left(R_{N} ; y, z\right)$ is complete and acyclic.

Definition 19. A preference profile $R_{N} \in X_{i \in N} \mathcal{R}_{i}$ satisfies sequential inclusion if for any pair $y, z \in A$ the binary relation $\succsim\left(R_{N} ; y, z\right)$ on $S\left(R_{N} ; y, z\right)$ is complete and acyclic. A domain $\times_{i \in N} \mathcal{R}_{i}$ satisfies sequential inclusion if any preference profile in this domain satisfies it.

Definition 20. For preferences $R_{i}, R_{i}^{\prime} \in \mathcal{R}_{i}$ and alternative $z \in A, R_{i}^{\prime}$ is a strict monotonic transformation of $R_{i}$ at $z$ if $R_{i}^{\prime}$ is such that for all $x \in A \backslash\{z\}$ such that $z R_{i} x, z P_{i}^{\prime} x$.

Definition 21. Let $R_{N}^{\prime}, R_{N} \in \mathrm{X}_{i \in N} \mathcal{R}_{i}$ be two preference profiles and let $z \in A$. We say that $R_{N}^{\prime}$ is a strict monotonic transformation of $R_{N}$ at alternative $z$ if for any $i \in N$, either $R_{i}^{\prime}=R_{i}$ or else $R_{i}^{\prime}$ is a strict monotonic transformation of $R_{i}$ at $z$.

Definition 22. A domain $X_{i \in N} \mathcal{R}_{i}$ satisfies indirect sequential inclusion if, for all profiles $R_{N} \in X_{i \in N} \mathcal{R}_{i}$, either (a) the profile $R_{N}$ satisfies sequential inclusion; or else (b) for each pair $y, z \in A$ there exists $R_{N}^{\prime} \in \mathrm{X}_{i \in N} \mathcal{R}_{i}$ where $R_{N \backslash S\left(R_{N} ; y, z\right)}^{\prime}=$ $R_{N \backslash S\left(R_{N} ; y, z\right)}$, such that

(1) $R_{N}^{\prime}$ is a strict monotonic transformation of $R_{N}$ at $z$,

(2) for any $i \in S\left(R_{N} ; y, z\right), y P_{i}^{\prime} z$, and

(3) $\succsim\left(R_{N}^{\prime} ; y, z\right)$ is complete and acyclic.

Let us mention that the examples in the following remark could be modified to encompass situations where agents may have different sets of individual preferences, any finite set of alternatives, and any number of agents when required.

Remark 1. Consider strict preferences. Intertwined and (indirect) sequential inclusion are independent.

Proof of Remark 1. Define a domain $\mathcal{R}_{1} \times \mathcal{R}_{2} \times \mathcal{R}_{3}, \mathcal{R}_{1}=\left\{R^{1}, R^{2}\right\}, \mathcal{R}_{2}=\left\{R^{3}, R^{4}\right\}$, and $\mathcal{R}_{3}=\left\{R^{5}, R^{6}\right\}$ where each $R^{l}$ is defined as in the example in the proof of part (3) of Proposition 1. Note that $\mathcal{R}$ is intertwined, but it violates sequential inclusion.

Let $R_{N}=\left(R^{1}, R^{4}, R^{6}\right)$ and $(y, z)=(\{a, b\}, \emptyset)$. Then, $\succsim\left(R_{N} ; y, z\right)$ on $S\left(R_{N} ; y, z\right)=\{2,3\}$ is not complete $\left(L\left(R^{4}, \emptyset\right) \nsubseteq\right.$ $\bar{L}\left(R^{6},\{a, b\}\right)$ and $\left.L\left(R^{6}, \emptyset\right) \nsubseteq \bar{L}\left(R^{4},\{a, b\}\right)\right)$. Note also that there is no strict monotonic transformation of $R_{N}$ at $z$ satisfying conditions (1)-(3) in Definition 22, thus indirect sequential inclusion is violated.

Consider now the following subdomain of single-peaked preferences over $A=\{x, y, z\}: \mathcal{P}=\left\{R^{1}, R^{2}, R^{3}\right\}$ such that

\begin{tabular}{ccc}
\hline$R^{1}$ & $R^{2}$ & $R^{3}$ \\
\hline \hline$x$ & $y$ & $z$ \\
$y$ & $x$ & $y$ \\
\cline { 2 - 3 }$z$ & $z$ & $x$ \\
\hline
\end{tabular}

This domain satisfies (indirect) sequential inclusion by Barberà et al. (2010). However, $\mathcal{P}$ is not intertwined since $R^{3}$ is not $(y, x)$-intertwined with $R^{1}$.

As we have just shown, in general, intertwinedness and indirect sequential inclusion are independent. However, in Proposition 6 below we show that any domain satisfying intertwinedness such that all agents have the same set of preferences do also satisfy indirect sequential inclusion. The converse does not hold as we have just shown in Remark 1 .

15 In what follows, and when this does not induce to error, we may omit the arguments $R_{N}, y$ and $z$ and just write $\succsim$. 
Proposition 6. Let $\mathrm{X}_{i \in N} \mathcal{P}_{i}$ be an intertwined domain such that $\mathcal{P}_{i}=\mathcal{P}_{j}$ for any $i, j \in N$. Then, $X_{i \in N} \mathcal{P}_{i}$ satisfies indirect sequential inclusion.

Proof. Let $R_{N} \in \mathrm{X}_{i \in N} \mathcal{P}_{i}$ and $x, y \in A$. Let $S\left(R_{N} ; x, y\right)=\left\{i \in N: x P_{i} y\right\}$. Suppose that $R_{N}$ violates sequential inclusion (otherwise, the proof ends). We show that $R_{N}$ satisfies indirect sequential inclusion. Take any order of the agents in $S\left(R_{N} ; x, y\right)$, without loss of generality let the order be $1,2,3, \ldots, \# S\left(R_{N} ; x, y\right)=k$. Take agents 1 and 2 . By intertwinedness, there exist $\bar{R}_{2}$ that is $y$-transform of $R_{2}$ and also an $x$-transform of $R_{1}$. Observe that $\bar{R}_{2}$ is such that $L\left(R_{2}, y\right) \subseteq \bar{L}\left(\bar{R}_{2}, y\right)$ and $x \bar{P}_{2} y$ (that is, $\bar{R}_{2}$ is a strict monotonic transformation of $R_{2}$ at $y$ according to Definition 6 in Barberà et al. (2010). Replace $R_{2}$ by $\bar{R}_{2}$ in $R_{N}$. By construction (by transitivity $x P_{1} y$ and $\bar{R}_{2}$ is an $x$-transform of $\left.R_{1}\right), L\left(R_{1}, y\right) \subseteq \bar{L}\left(\bar{R}_{2}, x\right)$. Thus, from now on take the new profile $\left(\bar{R}_{2}, R_{-2}\right)$. Observe that $S\left(\left(\bar{R}_{2}, R_{-2}\right) ; x, y\right)=S\left(R_{N} ; x, y\right)$ and $1 \succ 2$. Take agents 2 and 3 . By intertwinedness applied to $\bar{R}_{2}$ and $R_{3}$, there exists $\bar{R}_{3}$ that is $y$-transform of $R_{3}$ and also an $x$-transform of $\bar{R}_{2}$. Observe again that $\bar{R}_{3}$ is such that $L\left(R_{3}, y\right) \subseteq \bar{L}\left(\bar{R}_{3}, y\right)$ and $x \bar{P}_{3} y$ (that is, $\bar{R}_{3}$ is a strict monotonic transformation of $R_{3}$ at $y$ ). Replace $R_{3}$ by $\bar{R}_{3}$ in $\left(\bar{R}_{2}, R_{-2}\right)$. By construction (by transitivity $x P_{2} y$ and $\bar{R}_{3}$ is an $x$-transform of $\left.\bar{R}_{2}\right), L\left(R_{2}, y\right) \subseteq \bar{L}\left(\bar{R}_{3}, x\right)$, thus $2 \succ 3$. Note that $1 \succ 3$ since $L\left(R_{1}, y\right) \subseteq \bar{L}\left(\bar{R}_{2}, x\right)$ and $\bar{R}_{3}$ is an $x$-transform of $\bar{R}_{2}$ (i.e. $\left.\bar{L}\left(\bar{R}_{2}, x\right) \subseteq \bar{L}\left(\bar{R}_{3}, x\right)\right)$ thus $L\left(R_{1}, y\right) \subseteq \bar{L}\left(\bar{R}_{3}, x\right)$. Repeating the same argument for the remaining agents: 3 and 4,4 and 5 , and so on, we can construct a profile $R_{N}^{\prime} \equiv\left(R_{1}, \bar{R}_{2}, \ldots, \bar{R}_{n}\right)$ that satisfies conditions (1), (2), and (3) in part (b) of Definition 8 of indirect sequential inclusion in the paper Barberà et al. (2010). Thus, $X_{i \in N} \mathcal{P}_{i}$ satisfies indirect sequential inclusion.

As a corollary of Proposition 6 we obtain that the set of all lexicographically separable preferences violates intertwinedness since as shown in Barberà et al. (2010) this domain violates indirect sequential inclusion.

\section{Concluding remarks}

We have stressed the fact that the characteristics of the domains for which social choice functions are defined are crucial in determining what properties characterize these functions. In particular, we have shown that two very natural properties of social choice functions are equivalent to strategy-proofness for functions defined on intertwined domains.

Indeed, the two properties we propose (monotonicity and reshuffling invariance) are simple and attractive, and it is therefore very natural to ask under what conditions one can understand strategy-proofness as a simple consequence of such elementary requirements.

Although our requirement of intertwined domains is complex, we show it to be satisfied in several relevant instances, including some leading models for the allocation of public goods and also of private goods. For the environments where it holds, one can strictly identify those functions that satisfy monotonicity and reshuffling invariance with those that are strategy-proof.

We could have slightly altered our definition of intertwinedness in order to accommodate some further domains, like those of single-plateaued preferences. We could also have pushed further the implications of intertwined domains regarding the implementability of social choice functions. On that matter, let us just mention some straightforward results about implementation theory when combining our results with others in the literature. For any set of individuals preferences, strict or weak, by part (1) of Lemma 1 above and the revelation principle for dominant strategy implementation due to Gibbard (1973) stating that strategy-proofness is a necessary condition for dominant strategy implementation, our monotonicity and reshuffling invariance conditions are also necessary for dominant strategy implementation. More interestingly, when agents' preferences are intertwined and when the social choice function satisfies Saijo et al.'s (2007) weak non-bossiness, then monotonicity and reshuffling invariance are also sufficient for dominant strategy implementation (straightforward by our Theorem 1 and Theorem 3 in Saijo, Sjöstrom, and Yamato. Note that their weak non-bossiness trivially holds without indifferences).

But the main point is made: one should watch for the characteristics of domains. Only if these are rich enough it is possible to extend our intuitions, which are often based on the implicit assumption of universal domain, to other, more restricted contexts. We hope that these methodological points, as well as our examples and detailed results, can be helpful to researchers in the field.

\section{Appendix A}

In this appendix we show that intertwinedness and two domain conditions that turned out to be crucial to state interesting results in the social choice literature are independent. For lack of space we do not introduce such results and refer the interesting reader to each one of the papers.

Let us mention that all examples in the following remarks could be modified to encompass situations where agents may have different sets of individual preferences, any finite set of alternatives, and any number of agents when required.

\section{A.1. Intertwined versus connected in Kalai and Muller (1977)}

Some needed definitions are in order. 
Definition 23. We call a pair of distinct alternatives $x$ and $y$ trivial if there are no $R, R^{\prime} \in \mathcal{P}_{i}$ such that $x P y$ and $y P^{\prime} x$. Thus, the pair $(x, y)$ is trivial if there is always unanimity on it.

Definition 24. We say that $R$ and $R^{\prime} \in \mathcal{P}_{i}$ are connected if there exists a non-trivial pair $x, y \in A$ such that $x P y$ and $x P^{\prime} y$, i.e., if they agree on a non-trivial pair.

Definition 25. We say that $R$ and $R^{\prime} \in \mathcal{P}_{i}$ are indirectly connected if they are connected by a finite chain of connected preferences, i.e. there exist $Q_{1}, Q_{2}, \ldots, Q_{n} \in \mathcal{P}_{i}$ such that $R=Q_{1}, \ldots, Q_{n}=R^{\prime}$ and $Q_{i}$ is connected to $Q_{i+1}$ for $i=1,2, \ldots, n-1$.

A domain of preferences $\mathcal{P}_{i}$ is indirectly connected if any pair of individual preferences $R, R^{\prime} \in \mathcal{P}_{i}$ are indirectly connected.

Remark 2. Intertwined and indirectly connected domains are independent.

Proof of Remark 2. Let the domain $\chi_{i \in N} \mathcal{P}_{i}$ be such that for any $i \in N, \mathcal{P}_{i}=\left\{R^{1}, R^{2}\right\}$ where each $R^{l}$ is defined as in the example in the proof of part (3) of Proposition 1. We can check that this domain of preferences is intertwined. Note however that it is not (indirectly) connected since $R^{1}$ and $R^{2}$ are not connected (the only non-trivial pair is $(a, b)$ ).

Consider now the following subdomain of single-peaked preferences over $A=\{x, y, z\}: \mathcal{P}=\left\{R^{1}, R^{2}, R^{3}\right\}$ such that

\begin{tabular}{ccc}
\hline$R^{1}$ & $R^{2}$ & $R^{3}$ \\
\hline$x$ & $y$ & $z$ \\
\cline { 1 - 1 }$y$ & $x$ & $y$ \\
\cline { 2 - 3 }$z$ & $z$ & $x$ \\
\hline
\end{tabular}

Observe that $\mathcal{P}$ is indirectly connected: let the chain $Q_{1}=R^{1}, Q_{2}=R^{2}$ and $Q_{3}=R^{3}$ and observe that ( $x, z$ ) is a nontrivial pair for $Q_{1}, Q_{2}: R^{1}, R^{2}$ and $(y, x)$ is a non-trivial pair for $Q_{2}, Q_{3}: R^{2}, R^{3}$; thus, $\left(R^{1}, R^{2}\right)$ and $\left(R^{2}, R^{3}\right)$ are connected. However, $\mathcal{P}$ is not intertwined since $R^{3}$ is not $(y, x)$-intertwined with $R^{1}$.

\section{A.2. Intertwined versus linked in Aswal et al. (2003)}

Some previous notation follows: For any $R_{i} \in \mathcal{P}_{i}$, let $r_{1}\left(R_{i}\right)$ and $r_{2}\left(R_{i}\right)$ indicate the first and the second positioned alternative according to the preference $R_{i}$, respectively.

Definition 26. Alternatives $a_{j}, a_{k}$ are connected, say $a_{j} \sim a_{k}$, if $\exists R_{i}, R_{i}^{\prime} \in \mathcal{P}_{i}$ such that $r_{1}\left(R_{i}\right)=a_{j}, r_{2}\left(R_{i}\right)=a_{k}, r_{1}\left(R_{i}^{\prime}\right)=a_{k}$, and $r_{2}\left(R_{i}^{\prime}\right)=a_{j}$.

Definition 27. $B \varsubsetneqq A$ and $a_{j} \notin B . a_{j}$ is linked to $B$ if $\exists a_{k}, a_{r} \in B$ where $a_{j} \sim a_{k} \& a_{j} \sim a_{r}$.

Definition 28. $\mathcal{P}_{i}$ is linked if $\exists \sigma: A \rightarrow A$ one to one, such that (i) $a_{\sigma(1)} \sim a_{\sigma(2)}$, and (ii) $a_{\sigma(j)}$ is linked to $\left\{a_{\sigma(1)}, \ldots, a_{\sigma(j-1)}\right\}$, $j=3, \ldots, M$.

Remark 3. Intertwined and linked domains are independent.

Proof of Remark 3. The set of all single-peaked preferences $\mathcal{S}$ is intertwined (see Proposition 3). Aswal et al. (2003) show in their Example 3.4 that the domain $\mathcal{S}$ is not linked.

On the other hand, Aswal et al. (2003) define in their Example 3.1 the following linked domain: "Let $\mathcal{B}$ denote an individual set of preferences which has the following property: for all $a_{j}, a_{k} \in A$, there exists $R_{i} \in \mathcal{B}$ such that $r_{1}\left(R_{i}\right)=a_{j}$ and $r_{2}\left(R_{i}\right)=a_{k}$ ". Let us consider a particular example of $\mathcal{B}$ when $A=\{x, y, z, t\}$ :

\begin{tabular}{cccccccccccc}
\hline$R^{1}$ & $R^{2}$ & $R^{3}$ & $R^{4}$ & $R^{5}$ & $R^{6}$ & $R^{7}$ & $R^{8}$ & $R^{9}$ & $R^{10}$ & $R^{11}$ & $R^{12}$ \\
\hline$x$ & $z$ & $x$ & $y$ & $z$ & $y$ & $x$ & $t$ & $y$ & $t$ & $z$ & $t$ \\
$z$ & $x$ & $y$ & $x$ & $y$ & $z$ & $t$ & $x$ & $t$ & $y$ & $t$ & $z$ \\
$y$ & $t$ & $z$ & $t$ & $x$ & $t$ & $y$ & $z$ & $z$ & $x$ & $y$ & $x$ \\
$t$ & $y$ & $t$ & $z$ & $t$ & $x$ & $z$ & $y$ & $x$ & $z$ & $x$ & $y$ \\
\hline
\end{tabular}

Observe that $R^{1}$ is not $(x, z)$-intertwined with $R^{2}$. Thus $\mathcal{B}$ is linked but it is not intertwined. 


\section{References}

Alcalde-Unzu, J., Molis, E., 2011. Exchange of indivisible goods and indifferences: the top trading absorbing sets mechanism. Games Econ. Behav. 73, 1-16. Aswal, N., Chatterji, S., Sen, A., 2003. Dictatorial domains. Econ. Theory 22, 45-62.

Barberà, S., Beviá, C., 2002. Self-selection consistent functions. J. Econ. Theory 105, 263-277.

Barberà, S., Dutta, B., 1982. Implementation via protective equilibria. J. Math. Econ. 10, 49-65.

Barberà, S., Sonnenschein, H., Zhou, L., 1991. Voting by committees. Econometrica 59, 595-609.

Barberà, S., Gul, F., Stacchetti, E., 1993. Generalized median voter schemes and committees. J. Econ. Theory 61, 262-289.

Barberà, S., Jackson, M.O., Neme, A., 1997. Strategy-proof allotment rules. Games Econ. Behav. 18 (1), 1-21.

Barberà, S., Berga, D., Moreno, B., 2010. Individual versus group strategy-proofness: when do they coincide? J. Econ. Theory 145, 1648-1674.

Bogomolnaia, A., Deb, R., Ehlers, L., 2005. Strategy-proof assignment on the full preference domain. J. Econ. Theory 123, 161-186.

Cantala, D., 2004. Choosing a level of a public good when agents have and outside option. Soc. Choice Welfare 22, 491-514.

Chatterji, S., Sen, A., 2011. Tops-only domains. Econ. Theory 46, 255-282.

Ehlers, L., 2002a. Coalitional strategy-proof house allocation. J. Econ. Theory 105, 298-317.

Ehlers, L., 2002b. Multiple public goods and lexicographic preferences: replacement principle. J. Math. Econ. 37, 1-15.

Gibbard, A., 1973. Manipulation of voting schemes: a general result. Econometrica 41 (4), 587-601.

Jaramillo, P., Manjunath, V., 2011. The difference indifference makes in strategy-proof allocation of objects. CEDE documents, Universidad de los Andes.

Kalai, E., Muller, E., 1977. Characterization of domains admitting nondictatorial social welfare functions and nonmanipulable voting procedures. J. Econ. Theory $16,457-469$.

Maskin, E., 1985. The theory of implementation in Nash equilibrium: a survey. In: Hurwicz, Leonid, Schmeidler, David, Sonnenschein, Hugo (Eds.), Social Goals and Social Organization: Essays in Memory of Elisha Pazner. Cambridge University Press, pp. 173-204.

Maskin, E., 1999. Nash equilibrium and welfare optimality. Rev. Econ. Stud. 66, 23-38.

Miyagawa, E., 2001. Locating libraries on a street. Soc. Choice Welfare 18, 527-541.

Moulin, H., 1980. On strategy-proofness and single peakedness. Public Choice 35, 437-455.

Moulin, H., 1988. Axioms of Cooperative Decision Making. Econometric Society Monograph. Cambridge University Press, Cambridge.

Moulin, H., Shenker, S., 1992. Serial cost sharing. Econometrica 60, 1009-1037.

Muller, E., Satterthwaite, M.A., 1977. On the equivalence of strong positive association and strategy-proofness. J. Econ. Theory 14, 412-418.

Pápai, S., 2000. Strategy-proof assignment by hierarchical exchange. Econometrica 68, 1403-1433.

Roth, A.E., Sotomayor, M.A., 1990. Two-Sided Matching: A Study in Game-Theoretic Modeling and Analysis. In: Econometric Society Monographs, vol. 18. Cambridge University Press.

Saijo, T., Sjöstrom, S., Yamato, T., 2007. Secure implementation. Theoretical Econ. 2, 203-229.

Sato, S., 2010a. Circular domains. Rev. Econ. Design 14, 331-342.

Sato, S., 2010. A sufficient condition for the equivalence of strategy-proofness and nonmanipulability by preferences adjacent to the sincere one. Mimeo, Fukuoka University.

Sprumont, Y., 1991. The division problem with single-peaked preferences: a characterization of the uniform allocation rule. Econometrica 59 (2), 509-519. 